\title{
FUBINI-GRIFFITHS-HARRIS RIGIDITY AND LIE ALGEBRA COHOMOLOGY*
}

\author{
JOSEPH M. LANDSBERG ${ }^{\dagger}$ AND COLLEEN ROBLES ${ }^{\dagger}$
}

\begin{abstract}
We prove a rigidity theorem for represented semi-simple Lie groups. The theorem is used to show that the adjoint variety of a complex simple Lie algebra $\mathfrak{g}$ (the unique minimal $G$ orbit in $\mathbb{P} \mathfrak{g}$ ) is extrinsically rigid to third order (with the exception of $\mathfrak{g}=\mathfrak{a}_{1}$ ).

In contrast, we show that the adjoint variety of $S L_{3} \mathbb{C}$ and the Segre product $S e g\left(\mathbb{P}^{1} \times \mathbb{P}^{n}\right)$ are flexible at order two. In the $S L_{3} \mathbb{C}$ example we discuss the relationship between the extrinsic projective geometry and the intrinsic path geometry.

We extend machinery developed by Hwang and Yamaguchi, Se-ashi, Tanaka and others to reduce the proof of the general theorem to a Lie algebra cohomology calculation. The proofs of the flexibility statements use exterior differential systems techniques.
\end{abstract}

Key words. Exterior differential systems, Lie algebra cohomology, projective rigidity, rational homogeneous varieties.

AMS subject classifications. 14M15, 14M17, 53C24, 53C30, 58A15.

\section{Introduction.}

1.1. History and statement of the motivating problem. This paper introduces new machinery to the study of exterior differential systems with symmetry. Our main result (Theorem 6.12 ) was motivated by the following question.

The problem of determining the projective (or extrinsic) rigidity of varieties $X \subset$ $\mathbb{C P}^{N}=\mathbb{P}^{N}$ dates back to Monge and has been studied by Fubini [8], Griffiths and Harris [9] and others. The problem may be stated informally as follows: given a homogeneous variety $Z=G / P \subset \mathbb{P} U=\mathbb{P}^{N}$ and an unknown variety $Y \subset \mathbb{P} W=\mathbb{P}^{M}$, how many derivatives do we need to take at a general point of $Y$ to determine whether or not $Y$ is projectively equivalent to $Z$ ? More precisely, there is a sequence of relative differential invariants of a projective variety $X \subset \mathbb{P}^{N}$, defined at a smooth point $x \in X$ (the Fubini forms, see $\S 3.1$ ) that encode the extrinsic geometric information of $X$. Let $T_{x} X$ and $N_{x} X=T_{x} \mathbb{P}^{N} / T_{x} X$ denote the tangent and normal spaces to $X$ at $x \in X$. Then the $k$-th Fubini form $F_{k, x}$ at $x$ is an element of $S^{k} T_{x}^{*} X \otimes N_{x} X$ modulo an equivalence relation. If we (locally) express $X$ as graph in $\mathbb{P}^{N}$ over its embedded tangent space, then the Fubini forms $F_{k, x}$ are defined by the degree $k$ coefficients in the Taylor series expansion of $X$ about $x$, modulo the equivalence relation given by other choices of first-order adapted local coordinates.

DeFinition 1.1. Let $X \subset \mathbb{P} U$ be a projective variety and let $x \in X$ be a general point.

- If $Y \subset \mathbb{P} W$ is another variety such that for a general point $y \in Y$, there exist bijective linear maps $\phi_{T}: T_{y}^{*} Y \rightarrow T_{x}^{*} X, \phi_{N}: N_{y} Y \rightarrow N_{x} X$, such that the induced linear maps

$$
\left(\phi_{T}\right)^{\circ \ell} \otimes \phi_{N}: S^{\ell} T_{y}^{*} Y \otimes N_{y} Y \rightarrow S^{\ell} T_{x}^{*} X \otimes N_{x} X
$$

take (equivalence classes of) Fubini forms of $Y$ to (equivalence classes of) Fubini forms of $X$ for all $\ell \leq k$, then we say that $Y$ agrees with $X$ to order $k$.

\footnotetext{
*Received March 7, 2011; accepted for publication November 25, 2011.

$\dagger$ Department of Mathematics, Texas A\&M University, Mailstop 3368 - Milner Hall, College Station, TX 77843-3368, USA (\{jml; robles $\} @$ math.tamu.edu).
} 
- We say $X$ is rigid at order $k$ if whenever a variety $Y$ agrees with $X$ to order $k$, there exists a linear map $\Phi: W \rightarrow U$ such that $\Phi(Y)=X$.

- When $X$ is not rigid at order $k$, but the set of distinct $Y$ agreeing with $X$ to order $k$ is finite dimensional, we say $X$ is quasi-rigid at order $k$.

- Otherwise we say $X$ is flexible at order $k$.

REMARK. In this paper we discuss the rigidity problem in $\mathbb{P} U$. It is also natural to study rigidity when the ambient space is a homogeneous variety $G^{\prime} / P^{\prime}$ and the model variety is a $G$-variety. See, for example, the study of Schubert varieties in compact Hermitian symmetric spaces in [3].

None of the examples in this paper are quasi-rigid. The paper [3] contains quasirigid examples for a closely related problem.

Projective space embedded as a linear subspace $\mathbb{P}^{n} \subset \mathbb{P}^{N}$ is easily seen to be rigid at order two. Fubini [8] proved that the smooth $n$-dimensional quadric hypersurface is rigid at order three when $n>1$. When $n=1$ Monge proved that the quadric is rigid at order five (cf. [15, Ex. 2.26]). Griffiths and Harris [9] conjectured that the Segre variety $\operatorname{Seg}\left(\mathbb{P}^{2} \times \mathbb{P}^{2}\right) \subset \mathbb{P}^{8}$ is rigid at order two, and the conjecture was proven in [16]. Then in [17] it was shown that any irreducible, rank two, compact Hermitian symmetric space (CHSS) in its minimal homogeneous embedding (except for the quadric hypersurface) is rigid at order two. Hwang and Yamaguchi [10] then solved the rigidity problem for all homogeneously embedded irreducible CHSS: other than the quadric hypersurface or $\mathbb{P}^{n}$, an irreducible, homogeneously embedded CHSS with osculating sequence of length $f$ is rigid at order $f$. (The length of the osculating filtration of a CHSS in its minimal homogeneous embedding is equal to its rank.)

The next simplest homogeneous varieties are the adjoint varieties $Z_{\text {ad }}^{G} \subset \mathbb{P} \mathfrak{g}$, the homogeneous complex contact manifolds. These are the unique closed orbits in $\mathbb{P} \mathfrak{g}$ of the adjoint action of the associated complex simple Lie group $G$. When $G=C_{n}$, $Z_{\mathrm{ad}}^{C_{n}}=v_{2}\left(\mathbb{P}^{2 n-1}\right)$ is the quadratic Veronese variety which is CHSS for the larger group $A_{2 n-1}$. In [16] $v_{2}\left(\mathbb{P}^{m}\right), m>1$, was shown to be rigid at order three. This variety is flexible at order two since its second fundamental form is generic. The quadratic Veronese varieties have vanishing third and fourth order Fubini forms. In [22] it was observed that the third and fourth order Fubini invariants are non-zero for the other adjoint varieties. This led the authors to speculate that the other adjoint varieties would be rigid at order four, but not three. Robles [23] then showed that $Z_{\mathrm{ad}}^{A_{n}}, n>1$, is rigid at order three. (Note that, with the exceptions of $\mathfrak{a}_{n}$ and $\mathfrak{c}_{n}$, the adjoint representations of the complex simple Lie algebras are fundamental.)

\subsection{Results.}

ThEOREM 1.2. The adjoint variety of a complex simple Lie algebra $\mathfrak{g}, \mathfrak{g} \neq \mathfrak{a}_{1}$, is rigid at order three.

Theorem 1.2 is a consequence of Theorem $1.7\left(\mathfrak{g} \neq \mathfrak{a}_{n}\right.$ ), Lemma 7.3 (when $\mathfrak{g}=\mathfrak{a}_{n}$, $n>2$ ) and Proposition 5.2. The case of $A_{2}$ was resolved in [23] and we give a different proof in $\S 9.5$.

THEOREM 1.3. Assume $n, r>1$.

(a) The Veronese varieties $v_{d}\left(\mathbb{P}^{n}\right)$ are rigid at order $d+1$.

(b) The Veronese embeddings of the quadric hypersurfaces $v_{d}\left(Q^{n}\right)$ are rigid at order $2 d+1$

(c) For integers $1=a_{1} \leq a_{2} \leq \cdots \leq a_{r}$, the Segre variety $\operatorname{Seg}\left(\mathbb{P}^{a_{1}} \times \mathbb{P}^{a_{2}} \times \cdots \times \mathbb{P}^{a_{r}}\right)$ is rigid at order $r+1$. 
Theorem 1.3 is proven in $\S 10.1$. (The proof references calculations in $\S 7.3$.)

REMARK. If all the integers in Theorem 1.3 (c) satisfy $a_{i}>1$, then $S e g$ is rigid at order $r$, c.f. [10]. The third-order rigidity of $\operatorname{Seg}\left(\mathbb{P}^{1} \times \mathbb{P}^{1}\right) \subset \mathbb{P}^{3}$ is a consequence of Fubini's theorem [8], as it is a quadric hypersurface.

We apply standard exterior differential systems (EDS) techniques, guided by representation theory, to establish the following two flexibility results in $\S 8$ and $\S 9$, respectively. We remark that the calculations for Theorem 1.5 are quite long.

TheOREM 1.4. The Segre variety $\operatorname{Seg}\left(\mathbb{P}^{1} \times \mathbb{P}^{n}\right) \subset \mathbb{P}^{2 n+1}, n>1$, is flexible at order two. It is rigid at order three.

Theorem 1.4 is proved in $\S 8$. In [17] it was mistakenly remarked that $\operatorname{Seg}\left(\mathbb{P}^{1} \times \mathbb{P}^{n}\right)$ was rigid at order two. The reasons for this error are explained in $\S 8$. Notice that Theorem 1.4 implies that Theorem 1.3 (c) is sharp, and the rigidity statement is a consequence of Theorem 1.3 (c).

TheOREM 1.5. The adjoint variety $Z_{\mathrm{ad}}^{A_{2}}=$ Flag $_{1,2}\left(\mathbb{C}^{3}\right)$ is flexible at order two. The general integral manifold gives rise to a non-flat path geometry. There are also integral manifolds that are projectively inequivalent to the homogeneous model and give rise to flat path geometries.

Theorem 1.5 is proved in $\S 9$. We actually prove a stronger flexibility result. In $\S 4.2$ we define the $\left(I_{-1}, J_{-1}\right)$ system associated to any homogeneously embedded homogeneous variety. (This system is more restrictive than the second order Fubini system.) A parabolic $P \subset G$ determines a $\mathbb{Z}$-grading of $\mathfrak{g}$ and all $\mathfrak{g}$-modules, including $\mathfrak{g l}(U)$, where $G / P \subset \mathbb{P} U$. For $p=0,-1$, we define the $\left(I_{p}, J_{p}\right)$ exterior differential system by restricting the the component of the Maurer-Cartan form of $G L(U)$ taking values in the $q$-th homogeneous part of $\mathfrak{g l}(U)$ to take values in $\mathfrak{g} \subset \mathfrak{g l}(U)$, for $q \leq p$. See $\S 4.2$ for a detailed description.

While the $\left(I_{p}, J_{p}\right)$ systems are natural from the point of view of representation theory, except in the case of generalized cominuscule representations, they do not lead one to Lie algebra cohomology. To rectify this, we define a class of filtered EDS as follows: Define $\left(I_{p}^{\mathrm{f}}, \Omega\right)$ to be the $(p+1)$-filtered $E D S$ on $G L(U)$ with the same independence condition $\Omega$ as the $\left(I_{p}, J_{p}\right)$ system but only requiring integral manifolds $i: M \rightarrow G L(U)$ to satisfy $i^{*}\left(\omega_{\mathfrak{g}_{p-s}^{\perp}} \mid T^{-1-s} M\right)=0$ where the filtration $T^{-1} M \subset T^{-2} M \subset \cdots \subset T^{-k} M=\cdots=T M$ on $T M$ is induced from the grading on $T G L(U)$. See $\S 6$ for more details.

For the adjoint varieties, the $\left(I_{-1}, J_{-1}\right)$ system implies second order agreement plus partial third order agreement. The integral manifolds are bundles $\mathcal{F} \subset G L(U)$ over varieties $Y \subset \mathbb{P} U$. The EDS imposes the condition that the base varieties $Y$ all have contact hyperplane distributions, and that they support an intrinsic parabolic geometry. When $G=S L_{3} \mathbb{C}$ the parabolic geometry is the incidence space for a path geometry in the plane.

The variety $Z_{\mathrm{ad}}^{S L_{3}}=\operatorname{Flag}_{1,2}\left(\mathbb{C}^{3}\right)$ is the homogeneous model for the incidence space of a path geometry in the plane. This geometry has been studied extensively. The equivalence problem was solved by Cartan [6]. Intrinsically, on a three-fold $X$ equipped with two foliations by curves whose tangent lines span a contact distribution, there are two differential invariants, $J_{1}$ and $J_{2}$, which measure the failure of $X$ to be locally equivalent to the homogeneous model. We show that there are three distinct types of integral manifolds. The first class consists only of the standard homogeneous model. The second class depends (in the language of Cartan) on four functions of one 
variable. Despite this flexibility, all the examples in the second class are intrinsically flat. (One can think by analogy of the surfaces of zero Gauss curvature in Euclidean 3space, which depend on functions of one variable.) The third class of integral manifolds depends roughly on two functions of two variables. Here the intrinsic invariants are nonzero.

As mentioned above, the $\left(I_{-1}, J_{-1}\right)$ system is more restrictive than specifying second order agreement. In the case of the adjoint varieties it is less restrictive than specifying third order agreement. Theorems 1.2 and 1.3 are corollaries of Theorem 1.7 below. Loosely speaking, adjoint varieties for simple Lie algebras other than $\mathfrak{s l}_{2}$ and $\mathfrak{s l}_{3}$ are rigid to an order between two and three. In [22] it was observed that adjoint varieties are not rigid to order two, but only one non-isomorphic variety that agreed to order two was known: a "parabola" which had the same second fundamental form of the corresponding adjoint variety and all other differential invariants zero. These parabolas are not equipped with a contact structure.

Definition 1.6. A homogeneous variety $Z=G / P \subset \mathbb{P} U$ is rigid for the $\left(I_{p}, J_{p}\right)$ system (or rigid for the $\left(I_{p}^{\mathrm{f}}, \Omega\right)$ system that is defined in $\S 6$ ) if the only integral manifolds of the system on $S L(U)$ correspond to conjugates of $G \subset S L(U)$.

In this case the underlying projective varieties are all projectively equivalent to $Z$. Any variety rigid for the $\left(I_{p}, J_{p}\right)$ system (resp. the $\left(I_{p}^{\mathrm{f}}, \Omega\right)$ system) is automatically rigid for the $\left(I_{p+1}, J_{p+1}\right)$ system (resp. the $\left(I_{p+1}^{\mathrm{f}}, \Omega\right)$ system). Moreover, any variety rigid for the $\left(I_{p}^{\mathrm{f}}, \Omega\right)$ system is automatically rigid for the $\left(I_{p}, J_{p}\right)$ system.

Let $G$ have rank $r$, let $I \subset\{1, \ldots, r\}$ and write $P=P_{I} \subset G$ for the parabolic subgroup obtained by deleting negative root spaces corresponding to roots having a nonzero coefficient on any of the simple roots $\alpha_{i}, i \in I$.

THEOREM 1.7. Let $G$ be a complex semi-simple group and let $Z=G / P \subset \mathbb{P} U$ be a homogeneously embedded homogeneous variety (i.e., the orbit of a highest weight line in $\mathbb{P} U)$. Assume that no factor of $Z$ corresponds to a quadric hypersurface or $A_{n} / P_{I}$, with 1 or $n$ in $I$. Then $Z$ is rigid for the $\left(I_{-1}^{f}, \Omega\right)$-system.

The hypotheses above exclude the adjoint variety $Z_{\text {ad }}^{A_{n}}$, where $I=\{1, n\}$. Nonetheless,

THEOREM 1.8. The adjoint variety $Z_{\mathrm{ad}}^{A_{n}} \subset \mathbb{P}\left(\mathfrak{a}_{n}\right)$, for $n>2$, is rigid for the $\left(I_{-1}^{f}, \Omega\right)$-system.

Theorem 1.9. The Veronese variety $v_{d}\left(\mathbb{P}^{n}\right) \subset \mathbb{P}\left(S^{d} \mathbb{C}^{n+1}\right)=\mathbb{P} U_{d \omega_{1}}^{A_{n}}, n \geq 2$, and the adjoint variety $Z_{\mathrm{ad}}^{A_{2}} \subset \mathbb{P a}_{2}$ are rigid for the $\left(I_{0}^{f}, \Omega\right)$ system.

Theorems 1.7, 1.8 and 1.9 are proven in $\S 7.3$.

1.3. Reduction to Lie algebra cohomology. Given a homogeneous variety $Z=G / P \subset \mathbb{P} U=\mathbb{P}^{N}$ and a point $x \in Z$, one obtains two filtrations of $U$. The first is the osculating filtration (see $\S 4.1$ ) which any manifold in $\mathbb{P}^{N}$ has. This filtration corresponds to the spans of successive derivatives at $x$ of curves on $Z$. The second is the grading filtration which is given in terms of Lie algebra data (see §4.2). The two filtrations coincide if and only if $X$ is a homogeneously embedded compact Hermitian symmetric space (CHSS). There are natural exterior differential systems (EDS) on $G L(U)$ associated to each of these filtrations.

In [10], Hwang and Yamaguchi used methods of Tanaka and Se-ashi [25] as developed in [24] to establish an extrinsic rigidity result for CHSS subject to partial 
vanishing of the first Lie algebra cohomology groups. The methods of [10] were translated to the language of EDS in [18]. In brief, for CHSS, if one quotients the kernel of the Spencer differential by admissible normalizations in the fiber one arrives naturally at the Lie algebra cohomology group $H^{1}\left(\mathfrak{g}_{-}, \mathfrak{g}^{\perp}\right)$.

Two problems present themselves in generalizing beyond the case of CHSS. First, the Fubini systems and the EDS induced by the Lie algebra grading no longer coincide. Second, even if one ignores the Fubini system and restricts attention to the EDS induced by the Lie algebra gradings, the Spencer differential no longer coincides with the Lie algebra cohomology differential. We resolve the first problem for adjoint varieties by proving that integral manifolds of the third order Fubini system must also be integral manifolds of the $\left(I_{-1}, J_{-1}\right)$ system. In a planned sequel, we hope to resolve this problem for general $G / P$. (Resolving the problem in individual cases is straightforward, but tedious using our current technology.) The second difficulty is resolved in complete generality in this paper by introducing the filtered $\operatorname{EDS}\left(I_{p}^{\mathrm{f}}, \Omega\right)$ and simultaneously examining the torsion equations and prolongation in lowest nontrivial homogeneous degree (see our main Theorem 6.12):

The Spencer differential of the filtered system combined with the torsion equations in their first nontrivial homogenous degree $d$ is the degree $d$ homogeneous component of the Lie algebra cohomology differential $\partial^{1}$.

We hope this result will be useful in the study of other EDS with symmetry.

REMARK. The second order Fubini system induces the structure of a parabolic geometry on integral manifolds. When the grading on the tangent space is two-step, the third order Fubini system forces the parabolic geometry to be regular (e.g. one has a multi-contact structure on $T_{-1}$ ). The differential invariants that measure the failure of a parabolic geometry to be locally flat take values in the Lie algebra cohomology groups $H^{2}\left(\mathfrak{g}_{-}, \mathfrak{g}\right)$ (see, e.g., $\left.[4, \S 4]\right)$. It may be interesting to compare these intrinsic and extrinsic geometries and their invariants in greater detail.

1.4. Overview. In $\S 2$ we review basic definitions from exterior differential systems (EDS) and introduce the notion of reduced prolongations. In $\S 3$ we review the Fubini forms and introduce natural EDS (the Fubini systems) for studying rigidity of projective varieties. We then introduce the $\left(I_{0}, J_{0}\right)$ and $\left(I_{-1}, J_{-1}\right)$ EDS in $\S 4.2$. In $\S 5$ we show the $\left(I_{-1}, J_{-1}\right)$ system for adjoint varieties is implied by the third order Fubini system.

We define the filtered $\operatorname{EDS}\left(I_{p}^{\mathrm{f}}, \Omega\right)$ in $\S 6$. We then review Lie algebra cohomology and Kostant's theory in $\S 7.1$ and $\S 7.2$, respectively. In $\S 6$ we reduce proving rigidity for the $\left(I_{p}^{\mathrm{f}}, \Omega\right)$ system to proving that certain Lie algebra cohomology groups are zero. In $\S 7.3$ we identify groups and modules for which these cohomology groups vanish.

We establish flexibility of $\operatorname{Seg}\left(\mathbb{P}^{1} \times \mathbb{P}^{n}\right)$ and $Z_{\text {ad }}^{A_{2}}$ respectively in $\S 8$ and $\S 9$. In $\S 9.4$ we discuss the intrinsic geometry of the integral manifolds modeled on $Z_{\mathrm{ad}}^{A_{2}}$. Finally, in $\S 10$ we complete the proofs of the remaining rigidity results.

1.5. Generalizations. As discussed above, one could prove further rigidity results by having a better understanding of the Fubini forms. More precisely, one would need to determine to what order the Fubini form of a homogeneous variety must be specified in to fix the negative part of the Maurer-Cartan form in the Lie algebra grading. We plan to address this question in a subsequent paper.

All our systems have immediate generalizations to systems for homogeneous submanifolds $G / P \subset G^{\prime} / P^{\prime}$ of homogeneous varieties. (That is, $\mathbb{P} U$ may be replaced 
by the more general $G^{\prime} / P^{\prime}$.) Hong $[12,13]$ has results in this setting for three-step gradings. The machinery we have developed here will permit a more general study.

For the study of general Schubert varieties (see, for example, [3]), or more generally $G$-subvarieties of a homogeneous variety, there is another obstruction to applying Kostant's theory. Kostant's method of calculating $H^{1}(\mathfrak{n}, \Gamma)$ only applies if $\Gamma$ is a $\mathfrak{g}$ module and $\mathfrak{n} \subset \mathfrak{p} \subset \mathfrak{g}$. It may be possible to extend Kostant's theory to apply to systems for varieties modeled on Schubert varieties by finding a sufficiently invariant inner product on the relevant $\mathfrak{n}$ and $\Gamma$ to enable a "Hodge type" theorem.

1.6. Notational conventions. We work over the complex numbers throughout, all functions are holomorphic functions, manifolds complex manifolds etc... (although much of the theory carries over to $\mathbb{R}$, with some rigidity results even carrying over to the $C^{\infty}$ setting). In particular the notion of a general point of an analytic manifold makes sense, which is a point off of a finite union of analytic subvarieties. We use the labeling and ordering of roots and weights as in [1]. For subsets $X \subset \mathbb{P} V, \hat{X} \subset V$ denotes the corresponding cone. For a manifold $X, T_{x} X$ denotes its tangent space at $x$. For a submanifold $X \subset \mathbb{P} V, \hat{T}_{x} X \subset V$, denotes its affine tangent space. In particular, $T_{x} X=\hat{x}^{*} \otimes \hat{T}_{x} X / \hat{x}$. We use the summation convention throughout: indices occurring up and down are to be summed over. If $G$ is semi-simple of rank $r$, we write $P=P_{I} \subset G$ for the parabolic subgroup obtained by deleting negative root spaces corresponding to roots having a nonzero coefficient on any of the simple roots $\alpha_{i_{s}}, i_{s} \in I \subset\{1, \ldots, r\}$.

1.7. Acknowledgments. We thank R. Bryant, B. Doubrov, J. Hwang, S. Kumar, and K. Yamaguchi for useful discussions. The essential step for the transition to Lie algebra cohomology was resolved while A. Cap was visiting us. Remarkably Cap needed almost identical machinery for his work (see [5]) and, after a long day of discussions, both problems were resolved independently. This paper has benefitted tremendously from our conversations with him. In particular, the reformulation of our results in terms of filtered EDS was suggested by Cap.

2. Linear Pfaffian systems (EDS). A linear Pfaffian exterior differential system (EDS) on a manifold $\Sigma$ is a flag of subbundles of the cotangent bundle to $\Sigma, I \subset J \subset T^{*} \Sigma$ such that the map $I \rightarrow \Lambda^{2}\left(T^{*} \Sigma / J\right)$ given by $\theta \mapsto d \theta \bmod J$ is zero. An integral manifold is an immersed submanifold $i: N \rightarrow \Sigma$, where $n=\operatorname{dim} N=\operatorname{rank}(J / I)$, such that $i^{*}(I)=0$ and $i^{*}\left(\Lambda^{n}(J / I)\right)$ is non-vanishing.

A standard example is the space of one-jets $\Sigma=J^{1}(\mathbb{R}, \mathbb{R})$ with coordinates $(x, y, p)$ and $I=\{d y-p d x\} J=\{d y-p d x, d x\}$. Integral manifolds are the lifts $\left\{\left(x, f(x), f^{\prime}(x)\right)\right\}$ of graphs of arbitrary differentiable functions $f: \mathbb{R} \rightarrow \mathbb{R}$.

Fixing a general point $x \in \Sigma$ we set $V=(J / I)_{x}^{*}, W=I_{x}^{*}$. Calculating $d I \bmod I$ one defines the tableau $A \subset W \otimes V^{*}$ which (in the absence of torsion) essentially consists of the space of $n$-planes in $T_{x} \Sigma$ on which the forms in $I$ vanish; equivalently, the possible first order Taylor series of any integral manifold (expressed as a graph) through $x$. Because the systems in this paper are modeled on homogeneous spaces, $V, W$ and $A$ will be independent of the base point $x \in \Sigma$ : we suppress reference to it.

Calculating $d I \bmod I$ also yields the torsion, which is an element of $W \otimes \Lambda^{2} V^{*} / \tilde{\delta}\left(A \otimes V^{*}\right)$, where $\tilde{\delta}$ is the Spencer differential defined below. If there are no $n$-planes in $T_{x} \Sigma$ on which the forms in $I$ and their derivatives vanish at $x$, one says the system has torsion at $x$. In this case one must restrict to the submanifold $\Sigma^{\prime} \subset \Sigma$ where there is no torsion. 
Consider $f \in W \otimes V^{*}$ as a linear map $f: V \rightarrow W$ and define

$$
\tilde{\delta}:\left(W \otimes V^{*}\right) \otimes V^{*} \rightarrow W \otimes \Lambda^{2} V^{*}
$$

by $\tilde{\delta}(f \otimes \xi)=d f \wedge \xi$, and $A^{(1)}:=\left.\operatorname{ker} \tilde{\delta}\right|_{A \otimes V^{*}}$, the prolongation of $A$. Cartan developed a test to determine the "size" of the space of local integral manifolds by comparing a crude estimate for the dimension of the space of admissible second order terms in the Taylor series with the actual dimension, $\operatorname{dim} A^{(1)}$. When this test fails, one prolongs the system, defining a new system on $\Sigma \times A^{(1)}$.

The systems we will be dealing with all have at least one integral manifold, the corresponding homogeneous model. These systems may be grouped into three distinct categories.

DeFinition 2.1. We will say a system is rigid if all integral manifolds are equivalent to the homogeneous model. We will say a system is quasi-rigid if some prolongation of its tableau is zero and the system is not rigid. (In other words, some prolongation of the system is a Frobenius system.) In this case the space of local integral manifolds is finite dimensional. Otherwise we will say the system is flexible.

In the case that the system is flexible, the space of local integral manifolds is infinite dimensional - the integral manifolds will be determined by some space of free functions, as in the example of $J^{1}(\mathbb{R}, \mathbb{R})$ above.

The first derived system $I^{\prime} \subset I$ is the maximal subbundle such that $d I^{\prime} \equiv 0 \bmod I$ holds. We may and will suppress it in calculations. (See [11, p.216] or [2, p.45].)

A vector field $X \in \Gamma(T \Sigma)$ is a Cauchy characteristic vector field for the linear Pfaffian system $(I, J)$ if $\alpha(X)=0$ for all $\alpha \in I$. Cauchy characteristic vector fields correspond to (infinitesimal) symmetries of the EDS. By adding differential forms dual to the Cauchy characteristics to $J$, one obtains a new system whose integral manifolds are in natural correspondence with those of the original system. (See [11, p.205], [2, p.36].)

2.1. Reduced prolongations. In our case $\Sigma=G L(U)$, the group of invertible linear maps $U \rightarrow U$, and $I$ will consist of a component of the Maurer-Cartan form of $G L(U)$. We are interested in submanifolds of $\mathbb{P} U$; the integral manifolds of our EDS will be frame bundles $\mathcal{F} \subset G L(U)$ over these submanifolds. Consider the subgroup $\mathcal{N}^{\prime} \subset G L(U)$ that preserves the EDS: it will carry integral manifolds to integral manifolds. Let $\mathcal{N} \subset \mathcal{N}^{\prime}$ denote the subgroup that preserves the base submanifold in $\mathbb{P} U$.

The prolongation coefficients in $A^{(1)} \subset A \otimes V^{*}$ vary under the action of $\mathcal{N}$. These variations are the admissible normalizations of the prolongation coefficients. The admissible normalizations may be realized as the image of a map $\delta: \mathfrak{n} \rightarrow A \otimes V^{*}$, where $\mathfrak{n}$ is the Lie algebra of $\mathcal{N}$. We exploit this group action by defining a reduced prolongation of our EDS:

$$
A_{\text {red }}^{(1)}=A^{(1)} / \text { Image }\left(\delta: \mathfrak{n} \rightarrow A \otimes V^{*}\right) .
$$

As far as determining integral manifolds via the Cartan algorithm is concerned, normalizing by the action of $\mathcal{N}$ is of limited practical effect, but for the EDS discussed in this paper, the normalization corresponds to the denominator of a Lie algebra cohomology group. 


\section{The Fubini systems.}

3.1. The Fubini forms. Let $X^{n} \subset \mathbb{P} U=\mathbb{P}^{N}$ be a submanifold and let $x \in X$. Take linear local coordinates on $\mathbb{P} U$ about $x$ adapted so that $X$ is locally graphed over its (embedded) tangent plane at $x$. Expanding the functions in Taylor series, and collecting the homogeneous terms in each degree, one obtains a series of tensors $\underline{F}_{k}=\underline{F}_{k, X, x} \in\left(\hat{x}^{\otimes(k-1)}\right) \otimes S^{k} T^{*} X \otimes N_{x} X$. Here $N_{x} X=T_{x} \mathbb{P} U / T_{x} X$ denotes the normal space. If the local linear coordinates are $\left(x^{1}, \ldots, x^{n}, x^{n+1}, \ldots, x^{N}\right)=\left(x^{\alpha}, x^{\mu}\right)$ so that $X$ is locally given by equations $x^{\mu}=f^{\mu}\left(x^{\alpha}\right)$ then

$$
\underline{F}_{k, X, x}=(-1)^{k} \frac{\partial^{k} f^{\mu}}{\partial x^{i_{1}} \cdots \partial x^{i_{k}}} d x^{i_{1}} \circ \cdots \circ d x^{i_{k}} \otimes \frac{\partial}{\partial x^{\mu}} .
$$

The $\underline{F}_{k}$ depend on the choice of linear coordinates. To rectify this ambiguity, let $\pi: \mathcal{F}^{1} \rightarrow X$ denote the bundle of first order adapted frames, that is

$$
\mathcal{F}^{1}=\left\{\left(e_{0}, \ldots, e_{N}\right) \in G L(U) \mid\left[e_{0}\right] \in X, \hat{T}_{\left[e_{0}\right]} X=\operatorname{span}\left\{e_{0}, \ldots, e_{n}\right\}\right\} \subset G L(U) .
$$

Here the $e_{A}$ are to be considered as column vectors. The fiber over $x$ is isomorphic to the space of first order adapted linear coordinates based at $x$. Thus one obtains well defined $F_{k} \in \Gamma\left(\mathcal{F}^{1}, \pi^{*}\left(L^{k-1} \otimes S^{k} T^{*} X \otimes N X\right)\right)$ which are called the Fubini forms [11]. Here $L$ is the line bundle $\mathcal{O}_{\mathbb{P} U}(-1)$, the bundle with fiber $L_{x}=\hat{x}$. We use the notation $L^{k}:=L^{\otimes k}$. The Fubini forms determine $X$ up to projective equivalence.

3.2. The Maurer-Cartan form. Let $G$ be a Lie group, let $\omega \in \Omega^{1}(G, \mathfrak{g})$ denote the Maurer-Cartan form of $G$. By definition $\omega$ is the unique left-invariant differential form such that $\omega_{\text {Id }}: T_{\text {Id }} G \simeq \mathfrak{g} \rightarrow \mathfrak{g}$ is the identity map. It satisfies the Maurer-Cartan equation

$$
d \omega=-[\omega, \omega]
$$

To be precise, given two g-valued 1-forms $\rho$ and $\eta$, and two tangent vectors $u, v$, we define

$$
[\rho, \eta](u, v):=[\rho(u), \eta(v)]-[\rho(v), \eta(u)]
$$

In practice, $\rho$ and $\eta$ will be components (of a fixed homogeneous degree) of the MaurerCartan form.

If $G \subseteq G L(U)$ is a matrix Lie group, the Maurer-Cartan equation may be written $d \omega=-\omega \wedge \omega$ : if $\omega=\left(\omega_{B}^{A}\right)$, with $\omega_{B}^{A}$ the entries of the matrix, we have $(d \omega)_{B}^{A}=$ $-\omega_{C}^{A} \wedge \omega_{B}^{C}$. An essential feature of the Maurer-Cartan equation is that differentiation is reduced to an algebraic operation.

3.3. Second order Fubini systems. Fix vector spaces $L, T, N$ of dimensions $1, n, a$ and fix an element $F_{2} \in S^{2} T^{*} \otimes N \otimes L$. Let $U=L \oplus T \oplus N$, and let $\omega \in \Omega^{1}(G L(U), \mathfrak{g l}(U))$ denote the Maurer-Cartan form. Write $\mathfrak{g l}(U)=$ $(L \oplus T \oplus N)^{*} \otimes(L \oplus T \oplus N)$ and let, for example, $\omega_{L^{*} \otimes T}$ denote the component of $\omega$ taking values in $L^{*} \otimes T$. We have

$$
\omega=\left(\begin{array}{ccc}
\omega_{L^{*} \otimes L} & \omega_{T^{*} \otimes L} & \omega_{N^{*} \otimes L} \\
\omega_{L^{*} \otimes T} & \omega_{T^{*} \otimes T} & \omega_{N^{*} \otimes T} \\
\omega_{L^{*} \otimes N} & \omega_{T^{*} \otimes N} & \omega_{N^{*} \otimes N}
\end{array}\right)
$$


Writing the Maurer-Cartan equation component-wise yields, for example,

$$
d \omega_{L^{*} \otimes T}=-\omega_{L^{*} \otimes T} \wedge \omega_{L^{*} \otimes L}-\omega_{T^{*} \otimes T} \wedge \omega_{L^{*} \otimes T}-\omega_{N^{*} \otimes T} \wedge \omega_{L^{*} \otimes N} .
$$

Given $F_{2} \in L \otimes S^{2} T^{*} \otimes N$, the second order Fubini system for $F_{2}$ is

$$
I_{\mathrm{Fub}_{2}}=\left\{\omega_{L^{*} \otimes N}, \omega_{T^{*} \otimes N}-F_{2}\left(\omega_{L^{*} \otimes T}\right)\right\}, \quad J_{\mathrm{Fub}_{2}}=\left\{I_{\mathrm{Fub}_{2}}, \omega_{L^{*} \otimes T}\right\} .
$$

Its integral manifolds are submanifolds $\mathcal{F}^{2} \subset G L(U)$ that are adapted frame bundles of submanifolds $X \subset \mathbb{P} U$ having the property that at a point $x \in X$, the projective second fundamental form $F_{2, X, x}$ is equivalent to $F_{2}$. (The tautological system for frame bundles $\mathcal{F}^{1}$ of arbitrary $n$ dimensional submanifolds is given by $I=\left\{\omega_{L^{*} \otimes N}\right\}$, $J=\left\{I, \omega_{L^{*} \otimes T}\right\}$.)

Let $R \subset G L(L) \times G L(T) \times G L(N)$ denote the subgroup stabilizing $F_{2}$ and let

$$
\mathfrak{r} \subset\left(L^{*} \otimes L\right) \oplus\left(T^{*} \otimes T\right) \oplus\left(N^{*} \otimes N\right)=: \mathfrak{g l}(U)_{0, *}
$$

denote its subalgebra. These are the elements of $\mathfrak{g l}(U)_{0, *}$ annihilating $F_{2}$. (The motivation for the notation $\mathfrak{g l}(U)_{0, *}$ is explained in $\S 4$.1.) Since $\mathfrak{r}$ is reductive, we may decompose $\mathfrak{g l}(U)_{0, *}=\mathfrak{r} \oplus \mathfrak{r}^{\perp}$ as an $\mathfrak{r}$-module. In the notation of $\S 2$,

$$
V \simeq L^{*} \otimes T, \quad W \simeq\left(L^{*} \otimes N\right) \oplus\left(T^{*} \otimes N\right), \quad A \simeq \mathfrak{r}^{\perp},
$$

with $L^{*} \otimes N$ in the first derived system. That $\mathfrak{r}^{\perp} \subset V^{*} \otimes W$ may be seen as follows

$$
\begin{aligned}
d\left(\omega_{T^{*} \otimes N}-F_{2}\left(\omega_{L^{*} \otimes T}\right)\right)= & -\omega_{T^{*} \otimes L} \wedge \omega_{L^{*} \otimes N}-\omega_{T^{*} \otimes T} \wedge \omega_{T^{*} \otimes N} \\
& -\omega_{T^{*} \otimes N} \wedge \omega_{N^{*} \otimes N}+F_{2}\left(\omega_{L^{*} \otimes L} \wedge \omega_{L^{*} \otimes T}\right) \\
& +F_{2}\left(\omega_{L^{*} \otimes T} \wedge \omega_{T^{*} \otimes T}+\omega_{L^{*} \otimes N} \wedge \omega_{N^{*} \otimes T}\right) \\
\equiv & -\omega_{T^{*} \otimes T} \wedge F_{2}\left(\omega_{L^{*} \otimes T}\right)-F_{2}\left(\omega_{L^{*} \otimes T} \wedge \omega_{N^{*} \otimes N}\right. \\
& -F_{2}\left(-\omega_{L^{*} \otimes L} \wedge \omega_{L^{*} \otimes T}-\omega_{L^{*} \otimes T} \wedge \omega_{T^{*} \otimes T}\right) \bmod I \\
\equiv & \left(\omega_{0, *} \cdot F_{2}\right) \wedge \omega_{L^{*} \otimes T} \bmod I \\
\equiv & \left(\omega_{\mathbf{r}^{\perp}} \cdot F_{2}\right) \wedge \omega_{L^{*} \otimes T} \bmod I .
\end{aligned}
$$

Above, $\omega_{0, *} \cdot F_{2}$ denotes the action of the $\mathfrak{g l}(U)_{0, *}-$ valued component $\omega_{0, *}$ of the Maurer-Cartan form on $F_{2} \in S^{2} T^{*} \otimes N$. Recall that $\mathfrak{r}$ is the annihilator of this action. By definition $\omega_{0, *} \cdot F_{2}=\left(\omega_{\mathfrak{r}}+\omega_{\mathfrak{r}^{\perp}}\right) \cdot F_{2}=\omega_{\mathfrak{r}^{\perp}} \cdot F_{2}$.

3.4. Third order Fubini systems. Fix $F_{2} \in L \otimes S^{2} T^{*} \otimes N$ and $F_{3} \in$ $L^{2} \otimes S^{3} T^{*} \otimes N$. The third order Fubini system corresponding to $\left(F_{2}, F_{3}\right)$ is

$$
\begin{aligned}
& I_{\mathrm{Fub}_{3}}=\left\{\omega_{L^{*} \otimes N}, \omega_{T^{*} \otimes N}-F_{2}\left(\omega_{L^{*} \otimes T}\right), \omega_{\mathbf{r}^{\perp}} \cdot F_{2}-F_{3}\left(\omega_{L^{*} \otimes T}\right)\right\} \\
& J_{\mathrm{Fub}_{3}}=\left\{I_{\mathrm{Fub}_{3}}, \omega_{L^{*} \otimes T}\right\},
\end{aligned}
$$

where the last term in $I_{\mathrm{Fub}_{3}}$ is $N \otimes T^{*} \otimes T^{*} \otimes L$-valued, and $\omega_{\mathfrak{r}^{\perp}} . F_{2}$ is as in $\S 3.3$. Here

$$
V \simeq L^{*} \otimes T, \quad W \simeq\left(L^{*} \otimes N\right) \oplus\left(T^{*} \otimes N\right) \oplus \mathfrak{r}^{\perp},
$$

with the first two terms of $W$ in the first derived system. The tableau $A \simeq$ $\left(L \otimes T^{*}\right) \oplus\left(T \otimes N^{*}\right)$ sits in $W \otimes V^{*}$ by a calculation similar to (3.3).

Integral manifolds of the third order Fubini system are adapted frame bundles $\mathcal{F}^{3} \subset G L(U)$ over submanifolds of projective space whose second fundamental form is isomorphic to $F_{2}$ at each point and whose Fubini cubic is normalizable to $F_{3}$ at each point. Note that these systems admit reduced prolongations. The reductions (or normalizations) are by the subalgebra $\mathfrak{n} \subset\left(L \otimes T^{*}\right) \oplus\left(T \otimes N^{*}\right) \oplus\left(L \otimes N^{*}\right)$ such that $\mathfrak{n} .\left(\mathfrak{r}^{\perp} \cdot F_{2}-F_{3}\left(L^{*} \otimes T\right)\right)=0$.

In this paper we will be concerned with $\left(F_{2}, F_{3}\right)$ modeled on the Fubini forms of a homogeneous variety. 
4. A bi-grading of $\mathfrak{g l}(U)$.

4.1. The osculating filtration. Given a submanifold $X \subset \mathbb{P} U$, and $x \in X$, the osculating filtration at $x$

$$
U_{0} \subset U_{1} \subset \cdots \subset U_{\ell}=U
$$

is defined by

$U_{0}=\hat{x}, U_{1}=\hat{T}_{x} X, U_{2}=U_{1}+F_{2}\left(L^{*} \otimes S^{2} T_{x} X\right), \ldots, U_{r}=U_{\ell-1}+F_{\ell}\left(L^{* \ell-1} \otimes S^{\ell} T_{x} X\right)$.

The $F_{j}$ are the Fubini forms of $\S 3.1$. We may reduce the frame bundle $\mathcal{F}_{X}^{1}$ to framings adapted to the osculating sequence by specifying $e=\left(e_{0}, e_{\alpha}, e_{\mu_{2}}, \ldots, e_{\mu_{\ell}}\right) \in \mathcal{F}_{X}^{1}$ so that $U_{k}=\operatorname{span}\left\{e_{0}, e_{\alpha}, e_{\mu_{2}}, \ldots, e_{\mu_{k}}\right\}$. (The indices $\alpha$ and $\mu_{j}$ respectively range over $1, \ldots, n$ and $\operatorname{dim} U_{j-1}+1, \ldots, \operatorname{dim} U_{j}$.) From now on we work on this reduced framebundle, denoted $\mathcal{F}_{X}^{\ell} \subset \mathcal{F}_{X}^{1}$.

At each point of $\mathcal{F}_{X}^{\ell}$ we obtain a splitting of $U$. This induces a splitting

$$
\mathfrak{g l}(U)=\oplus \mathfrak{g l}(U)_{k, *} .
$$

The asterisk above is a place holder for a second splitting given (in $\S 4.2$ ) by the representation theory when $X=G / P$.

The osculating filtration of $U$ determines a refinement of the Fubini forms. Let $N_{k}=U_{k} / U_{k-1}$ and decompose $F_{s}=\oplus_{k} F_{k, s}$ so that $F_{k, s}: L^{* s-1} \otimes S^{s} T_{x} X \rightarrow N_{k}$. Although the Fubini forms do not descend to well-defined tensors on $X$, the fundamental forms $F_{k, k}$ do. By definition, $F_{k, k}: L^{* k-1} \otimes S^{k} T_{x} X \rightarrow N_{k, x} X$ is surjective.

REMARK. The only nonzero Fubini forms of a homogeneously embedded CHSS are the fundamental forms. For the adjoint varieties, the only nonzero Fubini forms are $F_{2,2}, F_{2,3}, F_{2,4}$.

4.2. The root grading and the $\left(I_{p}, J_{p}\right)$ systems . Let $\tilde{\mathfrak{g}}$ be a complex semisimple Lie algebra with a fixed set of simple roots $\left\{\alpha_{1}, \ldots, \alpha_{r}\right\}$, and corresponding fundamental weights $\left\{\omega_{1}, \ldots, \omega_{r}\right\}$. Let $I \subset\{1, \ldots, r\}$, and consider the irreducible representation $\mu: \tilde{\mathfrak{g}} \rightarrow \mathfrak{g r}(U)$ of highest weight $\lambda=\sum_{i \in I} \lambda^{i} \omega_{i}$. Set $\mathfrak{g}=\mu(\tilde{\mathfrak{g}})$, and let $\mu(G) \subset G L(U)$ be the associated Lie group so that $G / P \subset \mathbb{P} U$ is the orbit of a highest weight line. Write $P=P_{I} \subset G$ for the parabolic subgroup obtained by deleting negative root spaces corresponding to roots having a nonzero coefficient on any of the simple roots $\alpha_{i}, i \in I$.

Since $\tilde{\mathfrak{g}}$ is reductive, we have a splitting $\mathfrak{s l}(U)=\mathfrak{g} \oplus \mathfrak{g}^{\perp}$, where $\mathfrak{g}^{\perp}$ is the $\tilde{\mathfrak{g}}$ submodule of $\mathfrak{g l}(U)$ complementary to $\mathfrak{g}$. Let $\omega \in \Omega^{1}(G L(U), \mathfrak{g l}(U))$ denote the Maurer-Cartan form of $G L(U)$, and let $\omega_{\mathfrak{g}}$ and $\omega_{\mathfrak{g}^{\perp}}$ denote the components of $\omega$ taking values in $\mathfrak{g}$ and $\mathfrak{g}^{\perp}$, respectively.

The bundle $\mathcal{F}_{G / P}^{\ell}$ admits a reduction to a bundle $\mathcal{F}_{G / P}^{G}=\mu(G)$. On this bundle the Maurer-Cartan form pulls-back to take values in $\mathfrak{g}$, that is, $\omega_{\mathfrak{g}^{\perp}}=0$. Conversely, all $\operatorname{dim}(G)$ dimensional integral manifolds of the system $I=\left\{\omega_{\mathfrak{g}^{\perp}}\right\}$ are conjugates of $\mu(G)$. That is, the $\omega_{\mathfrak{g}^{\perp}}=0$ system is rigid. Theorem 6.12 establishes the rigidity of weaker systems, subject to the partial vanishing of components of a Lie algebra cohomology group.

Let $Z=Z_{I} \subset \mathfrak{t}$ be the grading element corresponding to $\sum_{i \in I} \alpha_{i}$. The grading element $Z_{i}$ for a simple root $\alpha_{i}$ has the property that $Z_{i}\left(\alpha_{j}\right)=\delta_{j}^{i}$. In general $Z=$ 
$\sum_{i \in I} Z_{i}$. Thus, if $\left(c^{-1}\right)$ denotes the inverse of the Cartan matrix, then given a weight $\nu=\sum \nu^{j} \omega_{j}$

$$
Z(\nu)=\sum_{\substack{1 \leq j \leq r \\ i \in I}} \nu^{j}\left(c^{-1}\right)_{j, i} .
$$

The grading element induces a $\mathbb{Z}$-grading of $\mathfrak{g}=\oplus_{-k}^{k} \mathfrak{g} k$. To determine $k$ in the case $P=P_{\alpha_{i}}$ is a maximal parabolic, let $\tilde{\alpha}=\sum m_{j} \alpha_{j}$ denote the highest root; then $k=m_{i}$.

The module $U$ inherits a $\mathbb{Z}$-grading

$$
U=U_{Z(\lambda)} \oplus U_{Z(\lambda)-1} \oplus \cdots \oplus U_{Z(\lambda)-f} .
$$

The $U_{j}$ are eigen-spaces for $Z$. This grading is compatible with the action of $\tilde{\mathfrak{g}}$ : $\mu\left(\tilde{\mathfrak{g}}_{i}\right) . U_{j} \subset U_{i+j}$. We adopt the notational convention of shifting the grading on $U$ to begin at zero. The component $U_{0}$ (formally named $U_{Z(\lambda)}$ ) is one dimensional, corresponding to the highest weight line of $U$, and $G \cdot \mathbb{P} U_{0}=G / P \subset \mathbb{P} U$. (The labeling of the grading on $\mathfrak{g l}(U)=U^{*} \otimes U$ is independent of our shift convention.)

Note, in particular, that the vector space $\hat{T}_{[\mathrm{Id}]}(G / P) / \hat{\mathrm{Id}} \simeq \mathfrak{g} / \mathfrak{p}$ is graded from -1 to $-k$. The osculating grading on $U$ induces gradings of $\mathfrak{g l}(U), \mathfrak{g}$ and $\mathfrak{g}^{\perp}$.

We write

$$
\mathfrak{g l}(U)=\bigoplus_{s, j} \mathfrak{g l}(U)_{s, j}
$$

where the first index refers to the osculating grading (§4.1) induced by $G / P \subset \mathbb{P} U$ and the second the root grading. We adopt the notational convention

$$
\mathfrak{g l}(U)_{j}=\bigoplus_{s} \mathfrak{g l}(U)_{s, j}
$$

so if there is only one index, it refers to the root grading. Note that although $Z(\lambda)$ need not be an integer, $f$ is. So the grading of $\mathfrak{g l}(U)$ is indexed by integers $-f, \ldots, f$.

We began with frame bundles $\mathcal{F} \subset \mathrm{GL}(U)$. But rescaling $e_{0}$ we may assume that $\mathcal{F} \subset \mathrm{SL}(U)$, and we will do so from now on. We define the $\left(I_{p}, J_{p}\right)$ system on $S L(U)$ by $I_{p}=\left\{\omega_{\mathfrak{g}_{\leq p}^{\perp}}\right\}, J_{p}=\left\{I_{p}, \omega_{\mathfrak{g}_{-}}\right\}$. The EDS we will study are the filtered systems $\left(I_{p}^{f}, \Omega\right)$, which are weaker than the $\left(I_{p}, J_{p}\right)$ systems. (See $\S 6$.)

REMARK. The osculating grading coincides (up to a change of sign) with the Lie algebra grading if and only if $G / P$ is CHSS.

5. The adjoint varieties. In this section we describe the fundamental adjoint representations from a uniform perspective and prove that integral manifolds of the third order Fubini system for them are automatically integral manifolds for the corresponding $\left(I_{-1}, J_{-1}\right)$-system.

Remark. We have the following geometric models for adjoint varieties $Z_{\text {ad }}^{G}$.

○ $A_{n}=S L_{n+1} \mathbb{C}$ : The flag variety $\mathbb{F}_{1, n}\left(\mathbb{C}^{n+1}\right)$ of lines in hyperplanes in $\mathbb{C}^{n+1}$.

○ $C_{n}=S p_{2 n} \mathbb{C}$ : The Veronese variety $v_{2}\left(\mathbb{P}^{2 n-1}\right) \subset \mathbb{P}\left(S^{2} \mathbb{C}^{2 n}\right)$.

$\circ G=S O(n)$ : The variety $G_{Q}(2, n)$ of 2 -planes in $\mathbb{C}^{n}$ on which the quadratic form restricts to be zero. 
$\circ G=G_{2}$ : The variety $G_{\text {null }}(2, \operatorname{Im}(\mathbb{O}))$ of 2 -planes in the imaginary complexified octonions to which the octonionic multiplication restricts to be identically zero.

$\circ G=E_{6}$ : The variety of $\mathbb{P}^{6}$ 's on the Cayley plane.

The highest root $\tilde{\alpha}$ induces a five-step grading $\mathfrak{g}=\mathfrak{g}_{-2} \oplus \mathfrak{g}_{-1} \oplus \mathfrak{g}_{0} \oplus \mathfrak{g}_{1} \oplus \mathfrak{g}_{2}$. (The corresponding grading of $U=\tilde{\mathfrak{g}}$ is $U_{j}=\mathfrak{g}_{j-2}$.) The adjoint variety is the $G$-orbit of $\mathbb{P} \mathfrak{g}_{2}$.

Fix a Chevalley basis of $\mathfrak{g}$. Decompose $\mathfrak{g}_{0}=\mathfrak{f}+\mathbb{C}\left\{Z_{\tilde{\alpha}}\right\}$ into the semi-simple Levi factor $\mathfrak{f}$, and the one-dimensional (assuming $\mathfrak{g} \neq \mathfrak{a}_{n}$ ) $\mathbb{C}\left\{Z_{\tilde{\alpha}}\right\}$. In order to obtain the representation $\mathfrak{g}=\operatorname{ad}(\tilde{\mathfrak{g}}) \subset \mathfrak{g l}(U)$ we make the following observations. First, $Z_{\tilde{\alpha}}$ acts on $\mathfrak{g}_{j}$ by $j \mathrm{Id}$; and $\mathfrak{f}$ acts trivially on $\mathfrak{g}_{ \pm 2}$. Let $\widetilde{I I}^{-2}: \mathfrak{f} \rightarrow \mathfrak{g l}\left(\mathfrak{g}_{-1}\right)=$ $\mathfrak{g}_{-1} \otimes \mathfrak{g}_{-1}^{*}$ denote the action of $\mathfrak{f}$ on $\mathfrak{g}_{-1}$. (Our choice of notation is made to be compatible with the geometry that enters later.) The Killing form $B$ on $\mathfrak{g}$ allows us to identify $\mathfrak{g}_{ \pm 1} \simeq \mathfrak{g}_{\mp 1}{ }^{*}$, so that we may regard $\widetilde{I I}^{-2}$ as an element of $\mathfrak{f}^{*} \otimes \mathfrak{g}_{-1} \otimes \mathfrak{g}_{1}$. The bracket $[\cdot, \cdot]: \mathfrak{g}_{-1} \times \mathfrak{g}_{-1} \rightarrow \mathfrak{g}_{-2}$ induces a symplectic form $\Omega \in \Lambda^{2} \mathfrak{g}_{-1}^{*}$. The linear map $\mathfrak{g}_{-1} \rightarrow \mathfrak{g}_{-1}^{*}=\mathfrak{g}_{1}$ induced by $\Omega$ is an $\mathfrak{f}$-module isomorphism. (That is, the representation $\widetilde{I I}^{-2}$ is symplectic.) Let $I I^{-2}=\operatorname{contr}\left(B \otimes \widetilde{I I}^{-2} \otimes \Omega\right) \in \mathfrak{g}_{-1}^{*} \otimes \mathfrak{g}_{-1}^{*} \otimes \mathfrak{f}$ denote the natural contraction

$$
(\mathfrak{f} \otimes \mathfrak{f}) \otimes\left(\mathfrak{f}^{*} \otimes \mathfrak{g}_{-1}^{*} \otimes \mathfrak{g}_{-1}\right) \otimes\left(\mathfrak{g}_{-1}^{*} \otimes \mathfrak{g}_{-1}^{*}\right) \rightarrow \mathfrak{f} \otimes \mathfrak{g}_{-1}^{*} \otimes \mathfrak{g}_{-1}^{*}
$$

Because the representation $\widetilde{I I}^{-2}$ is symplectic, the image is in $\mathfrak{f} \otimes S^{2} \mathfrak{g}_{-1}^{*}$.

The canonical identification $\mathfrak{g}_{ \pm 1} \simeq \mathfrak{g}_{\mp 1}^{*}$ allows us to treat $I I^{-2}, \widetilde{I I}^{-2}$ and $\Omega$ as elements of $\mathfrak{f} \otimes S^{2} \mathfrak{g}_{1}, \mathfrak{f}^{*} \otimes \mathfrak{g}_{1}^{*} \otimes \mathfrak{g}_{1}$ and $\Lambda^{2} \mathfrak{g}_{1}$, respectively.

The adjoint variety admits a frame bundle $\mu(G)=\mathcal{F}_{\text {ad }}^{G} \subset \mathrm{GL}(U)$ on which the Maurer-Cartan form pulls-back to take values in $\mathfrak{g} \subset \mathfrak{g l}(U)$. Decomposed with respect to the bi-grading, $\omega$ takes the form

$$
\left(\begin{array}{cccccc}
2 Z_{\tilde{\alpha}} & \mathfrak{g}_{1} & \mathfrak{g}_{2} & 0 & 0 & 0 \\
\mathfrak{g}_{-1} & Z_{\tilde{\alpha}} \operatorname{Id}+\widetilde{I I}^{-2}(\mathfrak{f}) & \Omega\left(\mathfrak{g}_{1}\right) & \widetilde{I I}^{-2}\left(\Omega\left(\mathfrak{g}_{1}\right)\right) & -\frac{1}{2} \mathfrak{g}_{2} \operatorname{Id} & 0 \\
\mathfrak{g}_{-2} & -\frac{1}{2} \Omega\left(\mathfrak{g}_{-1}\right) & 0 & 0 & -\frac{1}{2} \mathfrak{g}_{1} & -\frac{1}{2} \mathfrak{g}_{2} \\
0 & I I^{-2}\left(\mathfrak{g}_{-1}\right) & 0 & \operatorname{ad}(\mathfrak{f}) & -I I^{-2}\left(\Omega\left(\mathfrak{g}_{1}\right)\right) & 0 \\
0 & -\mathfrak{g}_{-2} \mathrm{Id} & -\mathfrak{g}_{-1} & \widetilde{I I}^{-2}\left(\mathfrak{g}_{-1}\right) & -Z_{\tilde{\alpha}} \mathrm{Id}+\widetilde{I I}^{-2}(\mathfrak{f}) & \Omega\left(\mathfrak{g}_{1}\right) \\
0 & 0 & -2 \mathfrak{g}_{-2} & 0 & -\Omega\left(\mathfrak{g}_{-1}\right) & -2 Z_{\tilde{\alpha}}
\end{array}\right) .
$$

The last non-zero fundamental form is the second, so the osculating filtration has length two. The tangent space $T=N_{1}$ and the normal space $N=N_{2}$ decompose as $N_{1,-1} \oplus N_{1,-2}$ and $N_{2,-2} \oplus N_{2,-3} \oplus N_{2,-4}$, respectively, under the root grading. We write $T_{j}=N_{1, j}$ and $N_{j}=N_{2, j}$. Notice that $I I^{-2}$ is the restriction to $N_{-2}^{*}$ of $I I: N^{*} \rightarrow S^{2} T^{*}$. The induced bi-grading on $\mathfrak{g l}(U)_{\text {osc,alg }}$ is indicated in the table below.

\begin{tabular}{c||c|c|c|c|c|c} 
& $L^{*}$ & $T_{-1}{ }^{*}$ & $T_{-2}{ }^{*}$ & $N_{-2}{ }^{*}$ & $N_{-3}{ }^{*}$ & $N_{-4}{ }^{*}$ \\
\hline \hline$L$ & $(0,0)$ & $(-1,1)$ & $(-1,2)$ & $(-2,2)$ & $(-2,3)$ & $(-2,4)$ \\
\hline$T_{-1}$ & $(1,-1)$ & $(0,0)$ & $(0,1)$ & $(-1,1)$ & $(-1,2)$ & $(-1,3)$ \\
\hline$T_{-2}$ & $(1,-2)$ & $(0,-1)$ & $(0,0)$ & $(-1,0)$ & $(-1,1)$ & $(-1,2)$ \\
\hline$N_{-2}$ & $(2,-2)$ & $(1,-1)$ & $(1,0)$ & $(0,0)$ & $(0,1)$ & $(0,2)$ \\
\hline$N_{-3}$ & $(2,-3)$ & $(1,-2)$ & $(1,-1)$ & $(0,-1)$ & $(0,0)$ & $(0,1)$ \\
\hline$N_{-4}$ & $(2,-4)$ & $(1,-3)$ & $(1,-2)$ & $(0,-2)$ & $(0,-1)$ & $(0,0)$
\end{tabular}


Proposition 5.2. Every integral manifold of the third-order Fubini system $\left(I_{\mathrm{Fub}_{3}}, J_{\mathrm{Fub}_{3}}\right)$ for a given adjoint variety is an integral manifold of the $\left(I_{-1}, J_{-1}\right)$ system for the same adjoint variety.

Proof. Suppose that $\mathcal{F} \subset S L(U)$ is an integral manifold of the third-order Fubini system. We wish to show that the $\mathfrak{g}_{*,<0}^{\perp}$-valued component of the Maurer-Cartan form vanishes when pulled-back to $\mathcal{F}$. That the $\mathfrak{g}_{>0, *}^{\perp}$-valued component vanishes is an immediate consequence of the injectivity of the second fundamental form $F_{2}$ on each homogeneous component.

Referring to the table above, we see that there remain four blocks of the component of the Maurer-Cartan form in $\mathfrak{g}_{*,<0}^{\perp}$ to consider: the three $(0,-1)$ blocks $\omega_{T_{-2} \otimes T_{-1}^{*}}, \omega_{N_{-3} \otimes N_{-2}^{*}}$ and $\omega_{N_{-4} \otimes N_{-3}^{*}} ;$ and the singleton $(0,-2)$ block $\omega_{N_{-4} \otimes N_{-2}^{*}}$. The third Fubini form is defined by $(3.5)$ of $[11, \S 3.5]$. The vanishing of the $\mathfrak{g}^{\perp}$ component of the first two blocks is a consequence of the $S^{3} T_{-1}^{*} \otimes N_{-3}$ component of $F_{3}$. (This is the only nonzero component of $F_{3}$.) The vanishing of the $\mathfrak{g}^{\perp}$-component of the third and fourth blocks is given by the $S^{3}\left(T^{*}\right) \otimes N_{-4}$-component of $F_{3}$.

6. Filtered EDS. In [10], it was observed (in different language) that for rigidity problems associated to CHSS, the $\left(I_{-1}, J_{-1}\right)$ system could be proved to be rigid using Lie algebra cohomology. More precisely, the Spencer differential coincides with the Lie algebra cohomology differential $\partial_{1}^{1}$ and the admissible normalizations coincide with the image of the Lie algebra cohomology map $\partial_{1}^{0}$. This correspondence breaks down when $k>1$ (see Remark 6.7 below), but it can be restored with the use of filtered EDS and simultaneously studying the prolongation and torsion.

DEFINITION 6.1. Let $\Sigma$ be a manifold equipped with a filtration of its tangent bundle $T^{-1} \subset T^{-2} \subset \cdots \subset T^{-f}=T \Sigma$. Define an $r$-filtered Pfaffian EDS on $\Sigma$ to be a filtered ideal $I \subset T^{*} \Sigma$ whose integral manifolds are the immersed submanifolds $i: M \rightarrow \Sigma$ such that $\left.i^{*}\left(I_{u}\right)\right|_{i^{*}\left(T^{u-r}\right)}=0$ for all $u$, with the convention that $T^{-s}=T \Sigma$ when $-s<-f$.

Another way to view filtered EDS is to consider an ordinary EDS on the total space of the sum of the bundles $I_{u} \otimes\left(T \Sigma / T^{u+r}\right)$. In our case these bundles will be trivial with fixed vector spaces as models.

Define $\left(I_{p}^{\mathrm{f}}, \Omega\right)$ to be the $(p+1)$-filtered $\operatorname{EDS}$ on $G L(U)$ with filtered ideal $I_{p}^{\mathrm{f}}:=$ $\omega_{\mathfrak{g}_{\leq p}^{\perp}}$ and independence condition $\Omega$ given by the wedge product of the forms in $\omega_{\mathfrak{g}_{-}}$. We may view this as an ordinary EDS on

$$
G L(U) \times\left(\left[\mathfrak{g}_{p}^{\perp} \otimes\left(\mathfrak{g}_{-2} \oplus \cdots \oplus \mathfrak{g}_{-k}\right)^{*}\right] \oplus\left[\mathfrak{g}_{p-1}^{\perp} \otimes\left(\mathfrak{g}_{-3} \oplus \cdots \oplus \mathfrak{g}_{-k}\right)^{*}\right] \oplus \cdots \oplus\left[\mathfrak{g}_{p-k+2}^{\perp} \otimes \mathfrak{g}_{-k}^{*}\right]\right)
$$

where, giving $\mathfrak{g}_{i}^{\perp} \otimes \mathfrak{g}_{-j}^{*}$ linear coordinates $\lambda_{i, j}$, we have

$$
\begin{aligned}
I_{p}^{\mathrm{f}}=\{ & \omega_{\mathfrak{g}_{s}^{\perp}}, s \leq p-k+1 ; \quad \omega_{\mathfrak{g}_{p-k+2}^{\perp}}-\lambda_{p-k+2, k}\left(\omega_{\mathfrak{g}_{-k}}\right), \\
& \omega_{\mathfrak{g}_{p-k+3}^{\perp}}-\lambda_{p-k+3, k}\left(\omega_{\mathfrak{g}_{-k}}\right)-\lambda_{p-k+3, k-1}\left(\omega_{\mathfrak{g}_{-k+1}}\right), \ldots \\
& \left.\omega_{\mathfrak{g}_{p}^{\perp}}-\lambda_{p, k}\left(\omega_{\mathfrak{g}_{-k}}\right)-\cdots-\lambda_{p, 2}\left(\omega_{\mathfrak{g}_{-2}}\right)\right\} .
\end{aligned}
$$

We henceforth assume $\boldsymbol{p} \geq \mathbf{- 1}$. 
6.1. The $p=-1, k=2$ case. We give a proof of the main result in $\S 6.2$ below, but for the reader's convienence we work out the $p=-1$ and $k=2$ case explicitly to illustrate the central ideas.

Abbreviate

$$
\omega_{\mathfrak{s l}(U)_{s}}=: \omega_{s}
$$

Notice that $\mathfrak{g}_{s}^{\perp}=\mathfrak{s l}(U)_{s}$ for all $s \leq-3$. so that $\omega_{\mathfrak{g}_{s}^{\perp}}=\omega_{s}$, for all $s \leq-3$. Thus the ideal $I_{-1}^{\mathrm{f}}$ is

$$
I_{-1}^{\mathrm{f}}=\left\{\omega_{\mathfrak{g}_{-1}^{\perp}}-\lambda_{-1,2}\left(\omega_{\mathfrak{g}_{-2}}\right), \omega_{\mathfrak{g}_{-2}^{\perp}}, \omega_{-3}, \ldots, \omega_{-f}\right\}
$$

The calculations that follow utilize the Maurer-Cartan equation (3.1), and the facts that $[\mathfrak{g}, \mathfrak{g}] \subset \mathfrak{g}$ and $\left[\mathfrak{g}, \mathfrak{g}^{\perp}\right] \subset \mathfrak{g}^{\perp}$. It is easy to see that $\mathrm{d} \omega_{s} \equiv 0$ modulo $I_{-1}^{\mathrm{f}}$ when $s \leq-4$. Next, computing modulo $I_{-1}^{\mathrm{f}}$,

$$
\begin{aligned}
&-\mathrm{d} \omega_{-3} \equiv\left[\omega_{\mathfrak{g}_{-2}}, \omega_{\mathfrak{g}_{-1}^{\perp}}\right] \equiv\left[\omega_{\mathfrak{g}_{-2}}, \lambda_{-1,2}\left(\omega_{\mathfrak{g}_{-2}}\right)\right] \\
&-\mathrm{d} \omega_{\mathfrak{g}_{-2}^{\perp}} \equiv\left[\omega_{\mathfrak{g}_{-2}}, \omega_{\mathfrak{g}_{0}^{\perp}}\right]+\left[\omega_{\mathfrak{g}_{-1}}, \omega_{\mathfrak{g}_{-1}^{\perp}}\right]+\left[\omega_{\mathfrak{g}_{-1}^{\perp}}, \omega_{\mathfrak{g}_{-1}^{\perp}}\right]_{\mathfrak{g}^{\perp}} \\
& \equiv\left[\omega_{\mathfrak{g}_{-2}}, \omega_{\mathfrak{g}_{0}^{\perp}}\right]+\left[\omega_{\mathfrak{g}_{-1}}, \lambda_{-1,2}\left(\omega_{\mathfrak{g}_{-2}}\right)\right] \\
&+\left[\lambda_{-1,2}\left(\omega_{\mathfrak{g}_{-2}}\right), \lambda_{-1,2}\left(\omega_{\mathfrak{g}_{-2}}\right)\right]_{\mathfrak{g}^{\perp}} .
\end{aligned}
$$

Finally,

$$
\begin{aligned}
-\mathrm{d}\left(\omega_{\mathfrak{g}_{-1}^{\perp}}-\lambda_{-1,2}\left(\omega_{\mathfrak{g}_{-2}}\right)\right) \equiv \\
\quad\left[\omega_{\mathfrak{g}_{-2}}, \omega_{\mathfrak{g}_{1}^{\perp}}\right]+\left[\omega_{\mathfrak{g}_{-1}}, \omega_{\mathfrak{g}_{0}^{\perp}}\right]+\left[\omega_{\mathfrak{g}_{-1}^{\perp}}, \omega_{\mathfrak{g}_{0}}\right]+\left[\omega_{\mathfrak{g}_{-1}^{\perp}}, \omega_{\mathfrak{g}_{0}^{\perp}}\right]_{\mathfrak{g}^{\perp}} \\
+\mathrm{d} \lambda_{-1,2}\left(\wedge \omega_{\mathfrak{g}_{-2}}\right)-\lambda_{-1,2}\left(\left[\omega_{\mathfrak{g}_{-2}}, \omega_{\mathfrak{g}_{0}}\right]+\left[\omega_{\mathfrak{g}_{1}}, \omega_{\mathfrak{g}_{-1}}\right]+\left[\omega_{\mathfrak{g}_{-1}^{\perp}}, \omega_{\mathfrak{g}_{-1}^{\perp}}\right]_{\mathfrak{g}}\right) \\
\equiv \\
{\left[\omega_{\mathfrak{g}_{-2}}, \omega_{\mathfrak{g}_{1}^{\perp}}\right]+\left[\omega_{\mathfrak{g}_{-1}}, \omega_{\mathfrak{g}_{0}}\right]+\left[\lambda_{-1,2}\left(\omega_{\mathfrak{g}_{-2}}\right), \omega_{\mathfrak{g}_{0}}\right] } \\
+\left[\lambda-1,2\left(\omega_{\mathfrak{g}_{-2}}\right), \omega_{\mathfrak{g}_{0}^{\perp}}\right]_{\mathfrak{g}^{\perp}}+\mathrm{d} \lambda_{-1,2}\left(\wedge \omega_{\mathfrak{g}_{-2}}\right) \\
+\lambda_{-1,2}\left(\left[\omega_{\mathfrak{g}_{-2}}, \omega_{\mathfrak{g}_{0}}\right]+\left[\omega_{\mathfrak{g}_{-1}}, \omega_{\mathfrak{g}_{-1}}\right]+\left[\lambda_{-1,2}\left(\omega_{\mathfrak{g}_{-2}}\right), \lambda_{-1,2}\left(\omega_{\mathfrak{g}_{-2}}\right)\right]_{\mathfrak{g}}\right)
\end{aligned}
$$

Here the bracket is as indicated in $\S 3.2$ and $[\cdot, \cdot]_{\mathfrak{g}}\left(\operatorname{resp} .[\cdot, \cdot]_{\mathfrak{g}^{\perp}}\right)$ denotes the component of the bracket taking values in $\mathfrak{g}$ (resp. $\mathfrak{g}^{\perp}$ ).

The three differentials above must vanish on an integral element modulo $I_{-1}^{\mathrm{f}}$. Notice that the vanishing of (6.4) implies

$$
\omega_{\mathfrak{g}_{0}^{\perp}}=\lambda_{0,1}\left(\omega_{\mathfrak{g}_{-1}}\right)+\lambda_{0,2}\left(\omega_{\mathfrak{g}_{-2}}\right)
$$

for some $\lambda_{0, j} \in \mathfrak{g}_{0}^{\perp} \otimes \mathfrak{g}_{-j}^{*}$.

Now consider the degree one $(1=p+2)$ homogeneous component of $(6.3,6.4,6.5)$, simultaneously examining the torsion and tableau. In order the have an integral element, $\lambda_{1}:=\oplus_{s=-1}^{0} \lambda_{s, 1-s}$ must be in the kernel of the map

$$
\delta_{1}: \oplus_{s=-1}^{0}\left(\mathfrak{g}_{s}^{\perp} \otimes \mathfrak{g}_{-s-1}^{*}\right) \rightarrow\left(\mathfrak{g}_{-1}^{\perp} \otimes \mathfrak{g}_{-1}^{*} \wedge \mathfrak{g}_{-1}^{*}\right) \oplus\left(\mathfrak{g}_{-2}^{\perp} \otimes \mathfrak{g}_{-1}^{*} \wedge \mathfrak{g}_{-2}^{*}\right)
$$


defined as follows. Given $u_{-1}, v_{-1} \in \mathfrak{g}_{-1}$,

$$
\delta_{1}\left(\lambda_{1}\right)\left(u_{-1} \wedge v_{-1}\right)=\left[\lambda_{0,1}\left(u_{-1}\right), v_{-1}\right]+\left[u_{-1}, \lambda_{0,1}\left(v_{-1}\right)\right]-\lambda_{-1,2}\left(\left[u_{-1}, v_{-1}\right]\right) .
$$

For $u_{-1} \in \mathfrak{g}_{-1}, v_{-2} \in \mathfrak{g}_{-2}$

$$
\delta_{1}\left(\lambda_{1}\right)\left(u_{-1} \wedge v_{-2}\right)=\left[\lambda_{0,1}\left(u_{-1}\right), v_{-2}\right]+\left[u_{-1}, \lambda_{-1,2}\left(v_{-2}\right)\right] .
$$

That is, $\delta_{1}=\partial_{1}^{1}$, where $\partial_{1}^{1}$ is the Lie algebra cohomology differential described in $\S 7$.

REMARK 6.7. Note that had we instead used the $\left(I_{-1}, J_{-1}\right)$ system in equation (6.6), the $\lambda_{-1,2}$ term would be missing and we would not have $\partial_{1}^{1}$. The map $\delta_{1}$ is akin to an 'augmented Spencer differential' it addresses both the torsion and prolongation in homogeneous degree 1.

Moreover, $\mathfrak{g}_{1}^{\perp}=\mathfrak{n} \cap \mathfrak{g l}(U)_{1}$ (cf. $\S 2.1$ ), and the Lie algebra cohomology denominator $\partial_{1}^{0}\left(\mathfrak{g}_{1}^{\perp}\right)$ is the space of admissible normalizations of the prolongation coefficients $\lambda_{1}$. Thus, the vanishing of $H_{1}^{1}\left(\mathfrak{g}_{-}, \mathfrak{g}^{\perp}\right)$ implies that normalized integral manifolds of the $\left(I_{-1}^{\mathrm{f}}, \Omega\right)$ system are in one to one correspondence with integral manifolds of the $\left(I_{0}^{\mathrm{f}}, \Omega\right)$ system.

\subsection{The general case. Define}

$$
\lambda_{s, *}:=\bigoplus_{j=p+2-s}^{k} \lambda_{s, j} \in \bigoplus_{j=p+2-s}^{k} \mathfrak{g}_{s}^{\perp} \otimes \mathfrak{g}_{-j}^{*},
$$

and set

$$
\lambda_{s, *}\left(\omega_{\mathfrak{g}_{<0}}\right):=\sum_{j=p+2-s}^{k} \lambda_{s, j}\left(\omega_{\mathfrak{g}_{-j}}\right) .
$$

Defining $\lambda_{s, j}=0$ when $s+j<p+2$ allows us to write the generators of the ideal $I_{p}^{\mathrm{f}}$ compactly as $\omega_{\mathfrak{g}_{s}^{\perp}}-\lambda_{s, *}\left(\omega_{\mathfrak{g}_{<0}}\right)$.

Observe that, if $s<-k$, then $\mathfrak{g}_{s}^{\perp}=\mathfrak{s l}(U)_{s}$, so that $\omega_{s} \equiv 0$ modulo $I_{p}^{\mathrm{f}}$. Also, 
$\omega_{\mathfrak{g}_{-k}^{\perp}} \equiv 0$ modulo $I_{p}^{\mathrm{f}}$. Now, the Maurer-Cartan equation (3.1) yields, modulo $I_{p}^{\mathrm{f}}$,

$$
\begin{aligned}
-2 \mathrm{~d}\left(\omega_{\mathfrak{g}_{s}^{\perp}}-\lambda_{s, *}\left(\omega_{\mathfrak{g}_{<0}}\right)\right) & \\
\equiv & \sum_{t+t^{\prime}=s}\left[\omega_{t}, \omega_{t^{\prime}}\right]_{\mathfrak{g}^{\perp}}+2 \mathrm{~d} \lambda_{s, *} \wedge \omega_{\mathfrak{g}_{<0}}+\lambda_{s, *}\left(\sum_{-k \leq t+t^{\prime}<-1}\left[\omega_{t}, \omega_{t^{\prime}}\right]_{\mathfrak{g}}\right) \\
\equiv & \sum_{t=-k}^{s-p-1}\left[\omega_{\mathfrak{g}_{t}}+\lambda_{t, *}\left(\omega_{\mathfrak{g}_{<0}}\right), \omega_{\mathfrak{g}_{s-t}}+\omega_{\mathfrak{g}_{s-t}^{\perp}}\right]_{\mathfrak{g}^{\perp}} \\
(6.8) \quad & +\sum_{t=s-p}^{p}\left[\omega_{\mathfrak{g}_{t}}+\lambda_{t, *}\left(\omega_{\mathfrak{g}_{<0}}\right), \omega_{\mathfrak{g}_{s-t}}+\lambda_{s-t, *}\left(\omega_{\mathfrak{g}_{<0}}\right)\right]_{\mathfrak{g}^{\perp}} \\
& +\sum_{t=p+1}^{s+k}\left[\omega_{\mathfrak{g}_{t}}+\omega_{\mathfrak{g}_{t}^{\perp}}, \omega_{\mathfrak{g}_{s-t}}+\lambda_{s-t, *}\left(\omega_{\mathfrak{g}_{<0}}\right)\right]_{\mathfrak{g}^{\perp}}+2 \mathrm{~d} \lambda_{s, *} \wedge \omega_{\mathfrak{g}_{<0}} \\
& \quad \sum_{j=p+2-s}^{k} \lambda_{s, j}\left(\sum_{t=-k}^{-j-p-1}\left[\omega_{\mathfrak{g}_{t}}+\lambda_{t, *}\left(\omega_{\mathfrak{g}_{<0}}\right), \omega_{\mathfrak{g}_{-j-t}}+\omega_{\mathfrak{g}_{-j-t}}\right]_{\mathfrak{g}}\right. \\
& +\sum_{t=-j-p}^{p}\left[\omega_{\mathfrak{g}_{t}}+\lambda_{t, *}\left(\omega_{\mathfrak{g}_{<0}}\right), \omega_{\mathfrak{g}_{-j-t}}+\lambda_{-j-t, *}\left(\omega_{\mathfrak{g}_{<0}}\right)\right]_{\mathfrak{g}} \\
& \left.+\sum_{t=p+1}^{k-j}\left[\omega_{\mathfrak{g}_{t}}+\omega_{\mathfrak{g}_{t}^{\perp}}, \omega_{\mathfrak{g}_{-j-t}}+\lambda_{-j-t, *}\left(\omega_{\mathfrak{g}_{<0}}\right)\right]_{\mathfrak{g}}\right)
\end{aligned}
$$

Observe that, for $s \leq-2 k, \mathrm{~d} \omega_{\mathfrak{g}_{s}^{\perp}} \equiv 0$ modulo $I_{p}^{\mathrm{f}}$; that is, for $s \leq-2 k, \omega_{\mathfrak{g}_{s}^{\perp}}$ lies in the first derived system $I^{\prime}$.

The necessary vanishing of (6.8) on integral elements tells us that there exist $\lambda_{s, j}$, taking values in $\mathfrak{g}_{s}^{\perp} \otimes \mathfrak{g}_{-j}{ }^{*}$, such that

$$
\omega_{\mathfrak{g}_{s}^{\perp}}=\sum_{j=1}^{k} \lambda_{s, j}\left(\omega_{\mathfrak{g}_{-j}}\right), \quad p+1 \leq s \leq p+k .
$$

The vanishing of (6.8) also imposes consraints on the $\omega_{\mathfrak{g}_{s}}$ and $\mathrm{d} \lambda_{s, *}$, but these terms will not appear when we consider the first non-zero component of the augmented Spencer differential $\delta_{p+2}$ below. (Of course, the $\omega_{\mathfrak{g}_{s}}, s \geq 0$, are dual to the Cauchy characteristics, and thus ought not influence the computation.)

Careful consideration of (6.8) shows that the right-hand side contains no components of homogeneous degree less than $p+2$. We identify the necessary condition that the degree $p+2$ homogeneous component of the right-hand side of (6.8) vanish on an integral element by evaluating the expression on $u \in T^{-i} / T^{1-i} \simeq \mathfrak{g}_{-i}$, 
$v \in T^{-j} / T^{1-j} \simeq \mathfrak{g}_{-j}$ with $s=(p+2)-(i+j), 1 \leq i, j \leq k$. Utilizing (6.9), we have

$$
\begin{aligned}
-2 \mathrm{~d}\left(\omega_{\mathfrak{g}_{s}^{\perp}}-\right. & \left.\lambda_{s, *}\left(\omega_{\mathfrak{g}_{<0}}\right)\right)(u, v) \\
\equiv & \sum_{t=-k}^{s-p-1}\left[\omega_{\mathfrak{g}_{t}}(u), \lambda_{s-t, *}\left(\omega_{\mathfrak{g}_{<0}}(v)\right)\right] \\
& +\sum_{t=s-p}^{p}\left[\omega_{\mathfrak{g}_{t}}(u), \lambda_{s-t, *}\left(\omega_{\mathfrak{g}_{<0}}(v)\right)\right]+\left[\lambda_{t, *}\left(\omega_{\mathfrak{g}_{<0}}(u)\right), \omega_{\mathfrak{g}_{s-t}}(v)\right] \\
& +\sum_{t=p+1}^{s+k}\left[\lambda_{t, *}\left(\omega_{\mathfrak{g}_{<0}}(u)\right), \omega_{\mathfrak{g}_{s-t}}(v)\right] \\
& -\lambda_{s, *}([\omega(u), \omega(v)]) \\
& {\left[\omega_{\mathfrak{g}}(u), \lambda_{s-i, *}\left(\omega_{\mathfrak{g}_{<0}}(v)\right)\right]-\left[\omega_{\mathfrak{g}}(v), \lambda_{s-j, *}\left(\omega_{\mathfrak{g}_{<0}}(u)\right)\right]-\lambda_{s, *}\left(\left[\omega_{\mathfrak{g}}(u), \omega_{\mathfrak{g}}(v)\right]\right) . }
\end{aligned}
$$

In particular, $\left(\lambda_{p+1,1}, \lambda_{p, 2}, \ldots, \lambda_{p+2-k, k}\right)$ must lie in the kernal of the map

$$
\delta_{p+2}: \bigoplus \mathfrak{g}_{p+2-m}^{\perp} \otimes \mathfrak{g}_{-m}^{*} \rightarrow \bigoplus \mathfrak{g}_{p+2-i-j}^{\perp} \otimes\left(\mathfrak{g}_{-i}^{*} \wedge \mathfrak{g}_{-j}^{*}\right)
$$

defined as follows: given $x \in \mathfrak{g}_{-i}$ and $y \in \mathfrak{g}_{-j}$,

$$
\begin{aligned}
& \delta_{p+2}\left(\lambda_{p+1,1}, \lambda_{p, 2}, \ldots, \lambda_{p+2-k, k}\right)(x, y) \\
& {\left[x, \lambda_{p+2-j, j}(y)\right]-\left[y, \lambda_{p+2-i, i}(x)\right]-\lambda_{p+2-i-j, i+j}([x, y]) .}
\end{aligned}
$$

As we will see below, $\delta_{p+2}=\partial_{p+2}^{1}$ is the degree $\mathrm{p}+2$ component of the first Lie algebra cohomology differential (described in $\S 7.1$ ). As in $\S 6.1, \delta_{p+2}$ should be viewed as the Spencer differential augmented to deal simultaneously with tableau and torsion.

Moreover, $\mathfrak{g}_{p+2}^{\perp}=\mathfrak{n} \cap \mathfrak{g l}(U)_{p+2}$ (cf. $\S 2.1$ ), and the Lie algebra cohomology denominator $\partial_{p+2}^{0}\left(\mathfrak{g}_{p+2}^{\perp}\right)$ is the space of admissible normalizations of the prolongation coefficients $\lambda_{p+2-m, m}$. Thus, the vanishing of $H_{p+2}^{1}\left(\mathfrak{g}_{-}, \mathfrak{g}^{\perp}\right)$ implies that normalized integral manifolds of the $\left(I_{p}^{\mathrm{f}}, \Omega\right)$ system are in one to one correspondence with integral manifolds of the $\left(I_{p+1}^{\mathrm{f}}, \Omega\right)$ system. We have established the following lemma.

LEMMA 6.11. Let $U$ be a complex vector space, and $\mathfrak{g} \subset \mathfrak{g l}(U)$ a represented complex semi-simple Lie algebra. Let $G / P \subset \mathbb{P} U$ be the corresponding homogeneous variety. Denote the induced $\mathbb{Z}$-gradings by $\mathfrak{g}=\mathfrak{g}_{-k} \oplus \cdots \oplus \mathfrak{g}_{k}$ and $U=U_{0} \oplus \cdots \oplus U_{-f}$. Fix an integer $p \geq-1$, and let $\left(I_{p}^{f}, \Omega\right)$ denote the filtered linear Pfaffian system given by (6.2).

If $H_{p+2}^{1}\left(\mathfrak{g}_{-}, \mathfrak{g}^{\perp}\right)=0$, then normalized integral manifolds of the $I_{p}^{f}$ system are in one to one correspondence with integral manifolds of the $I_{p+1}^{f}$ system.

Now a straightforward induction yields our main result.

THEOREM 6.12. Let $U$ be a complex vector space, and $\mathfrak{g} \subset \mathfrak{g l}(U)$ a represented complex semi-simple Lie algebra. Let $Z=G / P \subset \mathbb{P} U$ be the corresponding homogeneous variety (the orbit of a highest weight line). Denote the induced $\mathbb{Z}$-gradings by $\mathfrak{g}=\mathfrak{g}_{-k} \oplus \cdots \oplus \mathfrak{g}_{k}$ and $U=U_{0} \oplus \cdots \oplus U_{-f}$. Fix an integer $p \geq-1$, and let $\left(I_{p}^{f}, \Omega\right)$ denote the linear Pfaffian system given by (6.2). If $H_{d}^{1}\left(\mathfrak{g}_{-}, \mathfrak{g}^{\perp}\right)=0$, for all $d \geq p+2$, then the homogenous variety $G / P$ is rigid for the $\left(I_{p}^{f}, \Omega\right)$ system. 
7. The $\left(I_{p}^{\mathrm{f}}, \Omega\right)$-systems and Lie algebra cohomology.

7.1. Lie algebra cohomology. Let $\mathfrak{l}$ be a Lie algebra and let $\Gamma$ be an $\mathfrak{l}$-module. The maps

$$
\partial^{j}: \Lambda^{j} \mathfrak{l}^{*} \otimes \Gamma \rightarrow \Lambda^{j+1} \mathfrak{l}^{*} \otimes \Gamma
$$

are defined in a natural way to respect the Leibnitz rule [14], and give rise to a complex. Define $H^{k}(\mathfrak{l}, \Gamma):=\operatorname{ker} \partial^{k} / \operatorname{Image} \partial^{k-1}$. We will only have need of $\partial^{0}$ and $\partial^{1}$ which are as follows. If $X \in \Gamma$ and $v, w \in \mathfrak{l}$, then

$$
\partial^{0}(X)(v)=v \cdot X
$$

and if $\alpha \otimes X \in \Lambda^{1} \mathfrak{l}^{*} \otimes \Gamma$, then

$$
\partial^{1}(\alpha \otimes X)(v \wedge w)=\alpha([v, w]) X+\alpha(v) w \cdot X-\alpha(w) v \cdot X .
$$

Now let $\mathfrak{l}$ be a graded Lie algebra and $\Gamma$ a graded $\mathfrak{l}$-module. The chain complex and Lie algebra cohomology groups inherit gradings as well. Explicitly,

$$
\partial_{d}^{1}: \oplus_{i}\left(\mathfrak{l}_{-i}\right)^{*} \otimes \Gamma_{d-i} \rightarrow \oplus_{j \leq m}\left(\mathfrak{l}_{-j}\right)^{*} \wedge\left(\mathfrak{l}_{-m}\right)^{*} \otimes \Gamma_{d-j-m} .
$$

Taking $\mathfrak{l}=\mathfrak{g}_{-}, \Gamma=\mathfrak{g}^{\perp}$ and $d=p+2$, we obtain $\delta_{p+2}=\partial_{p+2}^{1}$ in the $I_{p}^{\mathrm{f}}$ system as asserted following (6.10). Moreover, the image of $\partial_{p+2}^{0}$ is the space of admissible normalizations.

7.2. Applying Kostant's theory. Kostant [14] shows that under the following circumstances one can compute $H^{k}(\mathfrak{l}, \Gamma)$ combinatorially:

1. $\mathfrak{l}=\mathfrak{n} \subset \mathfrak{p} \subset \mathfrak{g}$ is the nilpotent subalgebra of a parabolic subalgebra of a semi-simple Lie algebra $\mathfrak{g}$.

2. $\Gamma$ is a $\mathfrak{g}$-module.

Under these conditions, letting $\mathfrak{g}_{0} \subset \mathfrak{p}$ be the the (reductive) Levi factor of $\mathfrak{p}$, $H^{k}(\mathfrak{n}, \Gamma)$ is naturally a $\mathfrak{g}_{0}$-module. When $\Gamma$ is an irreducible $\mathfrak{g}$-module of highest weight $\lambda$, the irreducible $\mathfrak{g}_{0}$-modules appearing in $H^{k}(\mathfrak{n}, \Gamma)$ have highest weight $w .(\lambda)$, $w \in \mathcal{W}^{\mathfrak{p}}(k)$. Here, $\mathcal{W}^{\mathfrak{p}}(k)$ is a subset of the Weyl group of $\mathfrak{g}$, and $w \cdot(\lambda)=w(\lambda+\rho)-\rho$ denotes the affine action of $w$, with $\rho=\sum_{i} \omega_{i}=\frac{1}{2} \sum_{i} \alpha_{i}$ the sum of the fundamental weights of $\mathfrak{g}$.

For the $I_{p}^{\mathfrak{f}}$ system associated to $G / P_{I}$ we will only be concerned with $H^{1}\left(\mathfrak{g}_{-}, \mathfrak{g}^{\perp}\right)$. In this case $\mathcal{W}^{\mathfrak{p}}(1)$ is the set of simple reflections $\sigma_{i}$ corresponding to simple roots $\alpha_{i}$ such that $i \in I$. We will use the grading element $Z$, introduced in $\S 4.2$, to decompose the first cohomology group into homogeneous components $H^{1}\left(\mathfrak{g}_{-}, \mathfrak{g}^{\perp}\right)=$ $\bigoplus H_{d}^{1}\left(\mathfrak{g}_{-}, \mathfrak{g}^{\perp}\right)$.

Remark. Our $\mathfrak{g}_{-}$is Kostant's $\mathfrak{n}^{*}=\mathfrak{l}^{*}$. In particular, $H_{d}^{1}\left(\mathfrak{g}_{-}, \Gamma\right)=H_{-d}^{1}(\mathfrak{n}, \Gamma)$; and it is the latter that we shall be computing via Kostant.

7.3. Computing $\boldsymbol{H}_{\boldsymbol{d}}^{1}\left(\mathfrak{g}_{-}, \mathfrak{g}^{\perp}\right)$. By $\S 7.2$, we need only calculate the affine reflections in the simple roots $\alpha_{i}$ and to determine if they are nonzero in degree greater than $p+1$. We follow $[10,24]$ in this subsection.

Let $\sigma_{i_{0}}$ denote the affine reflection through simple root $\alpha_{i_{0}}, i_{0} \in I$. If we apply $\sigma_{i_{0}}$ to $\lambda=\lambda^{i} \omega_{i}$ we obtain, from Equation (4.1),

$$
Z\left(\sigma_{\alpha_{i_{0}}} . \lambda\right)=\sum_{i, s} \lambda^{i}\left(c^{-1}\right)_{i, i_{s}}-\lambda^{i_{0}}-1 .
$$


To see this, let $\langle\cdot, \cdot\rangle: \mathfrak{t}^{*} \times \mathfrak{t}^{*} \rightarrow \mathbb{C}$ be the pairing determined by $\left\langle\omega_{i}, \alpha_{j}\right\rangle=\delta_{i j}$.

$$
\begin{aligned}
Z\left(\sigma_{\alpha_{i_{0}}} \cdot \lambda\right) & =Z\left(\sigma_{\alpha_{i_{0}}}(\lambda+\rho)\right)-Z(\rho) \\
& =Z\left(\lambda+\rho-\left\langle\lambda+\rho, \alpha_{i_{0}}\right\rangle \alpha_{i_{0}}\right)-Z(\rho) \\
& =Z(\lambda)+Z(\rho)-\left\langle\lambda+\rho, \alpha_{i_{0}}\right\rangle Z\left(\alpha_{i_{0}}\right)-Z(\rho) \\
& =Z(\lambda)-\left\langle\lambda+\rho, \alpha_{i_{0}}\right\rangle \\
& =\sum_{i, s} \lambda^{i}\left(c^{-1}\right)_{i, i_{s}}-\left(\lambda_{i_{0}}+1\right) .
\end{aligned}
$$

In particular, for the trivial representation, $Z(\sigma .0)=-1$.

For positive weights $\lambda, Z\left(\sigma_{\alpha_{i_{0}}} \cdot \lambda\right)>-1$ when $\left(c^{-1}\right)_{i_{0}, i_{0}}>1$; and $Z\left(\sigma_{\alpha_{i_{0}}} \cdot \lambda\right) \geq-1$ when $\left(c^{-1}\right)_{i_{0}, i_{0}} \geq 1$. Recall, our $H_{d}^{1}\left(\mathfrak{g}_{-}, \Gamma\right)$ is Kostant's $H_{-d}^{1}(\mathfrak{n}, \Gamma)$ (cf. $\left.\S 7.2\right)$. Thus,

$H_{d}^{1}\left(\mathfrak{g}_{-}, \Gamma\right)=0$, for $d \geq 1$ (resp. $d>1$ ) when $\left(c^{-1}\right)_{i_{0}, i_{0}}>1$ (resp. $\geq 1$ ), for all $i_{0} \in I$.

The inverse Cartan matrices satisfy $\left(c^{-1}\right)_{j, j}>1$, except for the following cases (omitting redundancies):

$$
\begin{aligned}
& \left(c^{-1}\right)_{1,1}=\left(c^{-1}\right)_{n, n}=\frac{n}{n+1} \text { for } A_{n} \\
& \qquad \begin{array}{l}
\left(c^{-1}\right)_{2,2}=1 \text { for } A_{3} \\
\left(c^{-1}\right)_{1,1}=1 \text { for } B_{n}, n \geq 2, \text { and } D_{n}, n \geq 4 .
\end{array}
\end{aligned}
$$

By Schur's lemma, if $\mathfrak{g}$ is a semi-simple Lie algebra and $V$ is an irreducible $\mathfrak{g}$-module, then the trivial representation occurs exactly once in $\mathfrak{g l}(V)=V^{*} \otimes V$ and not at all in $\mathfrak{s l}(V)$. These observations, coupled with Theorem 6.12, allow us to deduce Theorem 1.7 and

THEOREM 7.2. Let $G$ be a complex semi-simple Lie group and let $G / P \subset \mathbb{P} U$ be a homogeneously embedded homogeneous variety. Assume that $G / P$ contains no factor corresponding a homogeneous variety $A_{n} / P_{I}$, with 1 or $n$ in $I$. Then, the $\left(I_{0}^{\mathrm{f}}, \Omega\right)$ system on $S L(U)$ is rigid.

Proof of Theorem 1.2. By Proposition 5.2 integral manifolds for the third-order Fubini system for adjoint varieties are also integral manifolds of the $\left(I_{-1}, J_{-1}\right)$ system and hence the $\left(I_{-1}^{\mathrm{f}}, \Omega\right)$ system. Theorem 1.2 now follows from Theorem 1.7 and Lemma 7.3 below.

Lemma 7.3. The $\left(I_{-1}^{f}, \Omega\right)$ system is rigid for $U=U_{\omega_{1}+\omega_{n}}^{A_{n}}, n>2$. The $\left(I_{0}^{f}, \Omega\right)$ system is rigid for $U=U_{\omega_{1}+\omega_{2}}^{A_{2}}$.

REMARK. This establishes Theorem 1.8.

Proof. We have the decomposition,

$$
\begin{aligned}
U^{*} \otimes U & =U_{\omega_{1}+\omega_{n}} \otimes U_{\omega_{1}+\omega_{n}} \\
& =U_{2\left(\omega_{1}+\omega_{n}\right)} \oplus U_{2 \omega_{1}+\omega_{n-1}} \oplus U_{\omega_{2}+2 \omega_{n}} \oplus U_{\omega_{2}+\omega_{n-1}} \oplus 2 U_{\omega_{1}+\omega_{n}} \oplus U_{0}
\end{aligned}
$$

So

$$
\mathfrak{g}^{\perp}=U_{2\left(\omega_{1}+\omega_{n}\right)} \oplus U_{2 \omega_{1}+\omega_{n-1}} \oplus U_{\omega_{2}+2 \omega_{n}} \oplus U_{\omega_{2}+\omega_{n-1}} \oplus U_{\omega_{1}+\omega_{n}}
$$


We calculate, assuming $n \geq 2$ (note that when $n=2, \omega_{n-1}=\omega_{1}$ )

$$
\begin{aligned}
Z\left(\sigma_{\alpha_{i_{0}}} \cdot 2\left(\omega_{1}+\omega_{n}\right)\right) & =1, \quad i_{0}=1, n \\
Z\left(\sigma_{\alpha_{1}} \cdot\left(2 \omega_{1}+\omega_{n-1}\right)\right) & =\left\{\begin{array}{cc}
0 & n \geq 3 \\
-1 & n=2
\end{array}\right. \\
Z\left(\sigma_{\alpha_{1}} \cdot\left(\omega_{2}+2 \omega_{n}\right)\right) & = \begin{cases}2 & n \geq 3 \\
2 & n=2\end{cases} \\
Z\left(\sigma_{\alpha_{1}} \cdot\left(\omega_{2}+\omega_{n-1}\right)\right) & = \begin{cases}1 & n \geq 3 \\
0 & n=2\end{cases} \\
Z\left(\sigma_{\alpha_{i_{0}}} \cdot\left(\omega_{1}+\omega_{n}\right)\right) & =0, \quad i_{0}=1, n .
\end{aligned}
$$

The $Z\left(\sigma_{\alpha_{n}} . \lambda\right)$ 's may be determined by the symmetry of the $A_{n}$ diagram. $\mathrm{Q}$

Now we consider some examples of the $\left(I_{0}^{f}, \Omega\right)$ system.

Example. Fubini's theorem. The third order Fubini system for a quadric hypersurface is equivalent to the $\left(I_{0}^{\mathrm{f}}, \Omega\right)$ system once one observes $\operatorname{Ann}(I I)=\mathfrak{g}_{0}+\mathbb{C} \subset$ $\mathfrak{g l}(U)_{0}$. We obtain a calculation free proof of Fubini's theorem.

Rigidity of the $\left(I_{0}^{\mathfrak{f}}, \Omega\right)$ system can only fail for $A_{n} / P_{I}$ if $\mathfrak{g}^{\perp}$ contains a module with large $\omega_{1}\left(\right.$ resp. $\omega_{n}$ ) coefficient and $1 \in I$ (resp. $n \in I$ ), and all other $\omega_{j}$ coefficients are relatively small. Theorem 1.9 is an example for which rigidity holds.

Proof of Theorem 1.9. For $v_{d}\left(\mathbb{P}^{n}\right) \subset \mathbb{P} S^{d} \mathbb{C}^{n+1}=\mathbb{P} U$ we consider $U \otimes U^{*}=$ $U_{d \omega_{1}} \otimes U_{d \omega_{n}}=\sum_{i=0}^{d} U_{i\left(\omega_{1}+\omega_{n}\right)}$. Hence $\mathfrak{g}^{\perp}=\sum_{i=2}^{d} U_{i\left(\omega_{1}+\omega_{n}\right)}$. We calculate $Z\left(\sigma_{\alpha_{1}} . i\left(\omega_{1}+\omega_{n}\right)\right)=i-1$.

A similar argument establishes the second half of the theorem for $U_{\omega_{1}+\omega_{2}}^{A_{2}}$. (The rigidity of $U_{\omega_{1}+\omega_{n}}^{A_{n}}, n>2$, is given by Lemma 7.3.)

We show in $\S 9$ that the nontrivial Lie algebra cohomology group $H_{1}^{1}$ that occurs from $Z\left(\sigma_{\alpha_{1}} .\left(3 \omega_{1}\right)\right)$ (here $\left.\omega_{n-1}=\omega_{1}\right)$ indeed makes the $\left(I_{-1}, J_{-1}\right)$ system flexible.

8. Flexibility of $\operatorname{Seg}\left(\mathbb{P}^{1} \times \mathbb{P}^{n}\right)$. Here we prove Theorem 1.4. Fix vector spaces $E$ and $F$ of dimensions 2 and $n+1$, respectively. Let $\operatorname{Seg}(\mathbb{P} E \times \mathbb{P} F) \subset \mathbb{P}(E \otimes F)$ denote the Segre variety. If $n=1$, then $\operatorname{Seg}\left(\mathbb{P}^{1} \times \mathbb{P}^{1}\right)$ is a quadric hypersurface; quadrics are flexible at order two and rigid at order three by Fubini's theorem [8]. So we assume for the rest of this section that $n>1$. We have already seen above that $\operatorname{Seg}\left(\mathbb{P}^{1} \times \mathbb{P}^{n}\right)$ is rigid to order three because it is rigid for the $\left(I_{0}, J_{0}\right)$ system.

We consider the $\left(I_{-1}, J_{-1}\right)$ system which agrees the second order Fubini system in this case. Here $W=\mathfrak{g}_{-1}^{\perp}, A=\mathfrak{g}_{0}^{\perp}$, and $V=\mathfrak{g}_{-1}$.

However, $H_{1}^{1}\left(\mathfrak{g}_{-}, \mathfrak{g}^{\perp}\right) \simeq \mathbb{C}^{n}$, which as a $\mathfrak{g}_{0}=\mathbb{C} \oplus\left(\mathfrak{s l}_{n}+\mathbb{C}\right)$ module is acted on by weights -4 for the first $\mathbb{C} \subset \mathfrak{s l}_{2}$ and 1 for the second $\mathbb{C} \subset \mathfrak{a}_{n}$ and is the dual of the standard representation for the $\mathfrak{s l}_{n}$ factor, corresponding to the weight $-4 \eta+\omega^{1}+\omega^{n}$ where $\eta$ is the fundamental weight of $\mathfrak{a}_{1}$ and $\omega_{j}$ are fundamental weights of $\mathfrak{a}_{n}$.

We now take our EDS to be the reduced prolongation. At this point the Spencer differential still coincides with the Lie algebra cohomology group, but the tableau no longer corresponds to a g-module, so we do not see any obvious way to use Kostant's theory. Fortunately in this case the calculation is straightforward and the prolonged system is involutive, with characters $\left(s_{1}, s_{2}, \ldots, s_{n+1}\right)=\left(n^{2}+2 n, 0, \ldots, 0\right)$. Moreover, it is not difficult to check that any $Y \subset \mathbb{P}^{2 n+1}$ agreeing with $S e g$ to second order admits a (reduced) frame bundle $\mathcal{F} \subset \mathrm{GL}_{2 n+2} \mathbb{C}$ on which the Maurer-Cartan form 
pulls-back to

$$
\left(\begin{array}{cccc}
\omega_{0}^{0} & \omega_{1}^{0} & \omega_{\beta}^{0} & r_{\beta}^{0} \omega_{0}^{1} \\
\omega_{0}^{1} & \omega_{1}^{1} & 0 & \omega_{\beta}^{0} \\
\omega_{0}^{\alpha} & r_{1}^{\alpha} \omega_{0}^{1} & \omega_{\beta}^{\alpha} & r \frac{\alpha}{\beta} \omega_{0}^{1}+\delta_{\beta}^{\alpha} \omega_{1}^{0} \\
0 & \omega_{0}^{\alpha} & \delta_{\beta}^{\alpha} \omega_{0}^{1} & \omega_{\beta}^{\alpha}+\delta_{\beta}^{\alpha}\left(\omega_{1}^{1}-\omega_{0}^{0}\right)
\end{array}\right) .
$$

Here $2 \leq \alpha \leq n+1$, and $\bar{\alpha}=\alpha+n$, and forms $\omega_{0}^{0}, \omega_{1}^{1}, \omega_{\beta}^{\alpha}, \omega_{0}^{1}, \omega_{0}^{\alpha}, \omega_{1}^{0}$ and $\omega_{\beta}^{0}$ are linearly independent. The $r_{1}^{\alpha}$ terms were introduced in the first reduced prolongation, and the $r_{\bar{\beta}}^{0}$ and $r \frac{\alpha}{\beta}$ coefficients in the second.

Given an integral manifold of the system, let $Y \subset \mathbb{P}^{2 n+1}$ denote the corresponding base manifold. By construction, $Y$ agrees with $\operatorname{Seg}(\mathbb{P} E \times \mathbb{P} F)$ to second order. Third order agreement holds if and only if the Fubini cubic vanishes on $Y$. This is equivalent to $r_{1}^{\alpha}=0$. This completes the proof of Theorem 1.4.

Remark. All (base) integral manifolds $Y$ of our system are ruled by $\mathbb{P}^{n}$ s. Consider the induced curve in the Grassmannian $\mathbb{G}\left(\mathbb{P}^{n}, \mathbb{P}^{2 n+1}\right)$. Such curves agree with the curve induced by $\operatorname{Seg}(\mathbb{P} E \times \mathbb{P} F)$ to order two, but differ with this curve at order three. In a remark in the introduction in [17] it was mistakenly stated that the above system was rigid, the error was primarily caused by the misconception that a curve in the Grassmannian would be determined by its second fundamental form.

9. The $\left(I_{-1}, J_{-1}\right)$ system for $A_{2}$. Here we consider $Y^{3} \subset \mathbb{P}^{7}$ with frame bundle $\mathcal{F}_{<0}$ on which the $\mathfrak{s l}\left(\mathfrak{a}_{2}\right)_{<0}$ component of the Maurer-Cartan form $\omega$ agrees with that of the $\mathfrak{s l}(3, \mathbb{C})$ adjoint variety $X$. Explicitly,

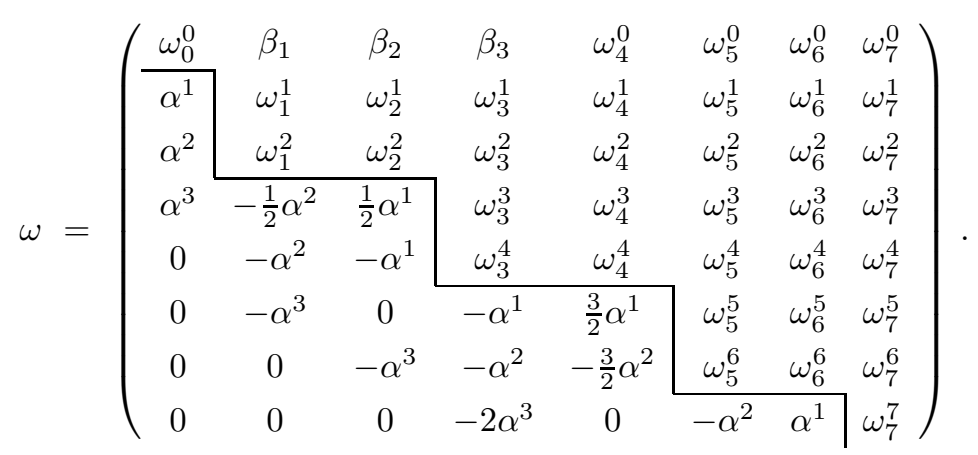

The $\omega_{a}^{0}=\beta_{a}$, are not assignments, merely renamings.

The quadrics of the second fundamental form are

$$
\begin{array}{ll}
q^{4}=-2 \alpha_{1} \alpha_{2} & q^{5}=-2 \alpha_{1} \alpha_{3} \\
q^{6}=-2 \alpha_{2} \alpha_{3} & q^{7}=-2 \alpha_{3}{ }^{2} .
\end{array}
$$

The tableau is torsion-free with characters $\left(s_{1}, s_{2}, s_{3}\right)=(17,4,0)$. In the course of prolonging, the 1-forms $\omega_{2}^{1}$ and $\omega_{1}^{2}$ are forced to be semi-basic (linear combinations of the $\left.\alpha^{a}\right)$. There exist rescalings of the $e_{j}, e=\left(e_{0}, \ldots, e_{7}\right) \in \mathcal{F}$, that preserve the EDS and scale the $\alpha^{2}$-coefficient of $\omega_{2}^{1}$ and the $\alpha^{1}$-coefficient of $\omega_{1}^{2}$ to be constant. We decompose the analysis into three cases: that the constants are $\{0,0\},\{0,1\}$ (two symmetric cases) or $\{1,1\}$.

9.1. Case 1. Assume that both constants are zero. Taking the second prolongation shows that $\omega$ satisfies the $\left(I_{0}, J_{0}\right)$ system. Thus, integral manifolds of $(9.1)$ are frame bundles over the adjoint variety $Z_{\mathrm{ad}}^{A_{2}}$. 
9.2. Case 2. Suppose that $\omega_{2}^{1} \equiv \alpha^{2} \bmod \alpha^{1}, \alpha^{3}$; and $\omega_{1}^{2} \equiv 0 \bmod \alpha^{2}, \alpha^{3}$. After restricting our parameter space (twice) to remove torsion, the second tableau has characters $\left(s_{1}, s_{2}, s_{3}\right)=(13,1,0)$. The (reduced) second prolongation is of dimension 9. Again, we must restrict the parameter space (twice, again) to obtain a torsionfree third tableau with characters $\left(s_{1}, s_{2}, s_{3}\right)=(9,1,0)$. The third prolongation has dimension 5. The fourth tableau (after one reduction for torsion) has characters $\left(s_{1}, s_{2}, s_{3}\right)=(4,0,0)$ and is involutive. The Maurer-Cartan form (the components of non-negative degree) is given below. In degree 0 (the block diagonal):

$\omega_{0}^{0}=f_{1} \alpha^{1}+f_{2} \alpha^{2}+\frac{1}{4} f_{1,2} \alpha^{3}+3 \omega_{1}^{1}, \quad \omega_{2}^{1}=\alpha^{2}, \quad \omega_{6}^{5}=\alpha^{2}-\frac{1}{2} f_{1} \alpha^{3}$

$0=\omega_{0}^{0}-\omega_{1}^{1}-\omega_{2}^{2}=\omega_{2}^{2}+\omega_{5}^{5}=\omega_{1}^{1}+\omega_{6}^{6}=\omega_{0}^{0}+\omega_{7}^{7}=\omega_{1}^{2}=\omega_{3}^{3}=\omega_{4}^{3}=\omega_{3}^{4}=\omega_{4}^{4}=\omega_{5}^{6}$.

Above the $f_{j}$ are functions on the (reduced) frame bundle, and the $f_{j, a}$ are the $\alpha^{a}-$ coefficients of $\mathrm{d} f_{j}$. In degree 1 :

$$
\begin{array}{ll}
\omega_{3}^{1}=\omega_{6}^{4}=\frac{1}{4} f_{1} \alpha^{2}-\beta_{2}, & \omega_{4}^{1}=\frac{9}{8} f_{1} \alpha^{2}-\frac{3}{2} \beta_{2}, \quad \omega_{6}^{3}=-\frac{1}{8} f_{1} \alpha^{2}-\frac{1}{4} f_{1,1} \alpha^{3}-\frac{1}{2} \beta_{2} \\
\omega_{7}^{5}=-\frac{1}{4} f_{1,1} \alpha^{3}-\beta_{2}, & \beta_{1}=\omega_{3}^{2}=-\frac{2}{3} \omega_{4}^{2}=-2 \omega_{5}^{3}=\omega_{5}^{4}=\omega_{7}^{6} .
\end{array}
$$

In degree 2:

$$
\begin{array}{lll}
\omega_{4}^{0}=-\frac{3}{8} f_{1,1} \alpha^{2}-\frac{3}{2} p_{1} \alpha^{3} & \omega_{5}^{1}=\frac{1}{8} f_{1,1} \alpha^{2}-\frac{3}{2} p_{1} \alpha^{3}-\frac{1}{2} \beta_{3} & \omega_{6}^{1}=\frac{1}{4} f_{1,2} \alpha^{2}+\frac{1}{4} f_{1,3} \alpha^{3}-\beta_{1} \\
\omega_{7}^{4}=p_{1} \alpha^{3}, \quad \omega_{5}^{2}=0 & \omega_{6}^{2}=-\frac{1}{8} f_{1,1} \alpha^{2}-\frac{3}{2} p_{1} \alpha^{3}-\frac{1}{2} \beta_{3} & \omega_{7}^{3}=-\frac{1}{8} f_{1,1} \alpha^{2}-6 p_{1} \alpha^{3}-\frac{1}{2} \beta_{3},
\end{array}
$$

with $p_{1}=\left(f_{1} f_{1,1}+f_{1,1,1}\right) / 24$, where $f_{1,1,1}$ is the $\alpha^{1}$-coefficient of $\mathrm{d} f_{1,1}$. Note that $p_{1}$ appears below in the Fubini cubic $r^{4}$. In degree 3:

$$
\begin{array}{ll}
\omega_{5}^{0}=-4 p_{1} \alpha^{2}-p_{3} \alpha^{3} & \omega_{6}^{0}=-p_{1} \alpha^{1}-\frac{1}{2} f_{1,3} \\
\omega_{7}^{1}=-p_{1} \alpha^{1}+\frac{1}{4} f_{1,3} \alpha^{2}+p_{2} \alpha^{3}+\frac{1}{4} f_{1} \beta_{1} & \omega_{7}^{2}=-p_{1} \alpha^{2}+p_{3} \alpha^{3},
\end{array}
$$

with $p_{2}=\left(f_{1,1} f_{1,2}+4 f_{1,1,3}\right) / 48$ and $p_{3}=\left(f_{1,1}^{2}-2 f_{1}^{2} f_{1,1}-2 f_{1} f_{1,1,1}\right) / 48$. And the final degree 41 -form is

$$
\omega_{7}^{0}=-4 p_{3} \alpha^{1}-4 p_{2} \alpha^{2}+p_{4} \alpha^{3}-\frac{1}{2} f_{1,1} \beta_{1},
$$

where $p_{4}$ is a polynomial in $f_{1}, f_{2}, f_{1, a}, f_{1,1,1}, f_{1,1,3}$ (homogeneous of degree 4 in derivatives).

The Fubini cubics are

$$
\begin{aligned}
r^{4}=-2 \alpha_{2}{ }^{3}+2 p_{1} \alpha_{3}{ }^{3} & r^{5}=3 \alpha_{1}{ }^{2} \alpha_{2}-\frac{3}{2} f_{1} \alpha_{2} \alpha_{3}{ }^{2}-\frac{1}{2} f_{1,1} \alpha_{3}{ }^{3} \\
r^{6}=-3 \alpha_{1} \alpha_{2}{ }^{2} & r^{7}=0 .
\end{aligned}
$$

These varieties are intrinsically flat (cf. $§ 9.4)$.

9.3. Case 3. Assume that $\omega_{2}^{1}=\alpha^{2} \bmod \alpha^{1}, \alpha^{3} ;$ and $\omega_{1}^{2}=\alpha^{1} \bmod \alpha^{2}, \alpha^{3}$. The second tableau (after two sets of restrictions on our parameter space to remove torsion) has characters $\left(s_{1}, s_{2}, s_{3}\right)=(15,2,0)$. The second (reduced) prolongation has dimension 14. The third tableau (after one set of restrictions to remove torsion) has characters $\left(s_{1}, s_{2}, s_{3}\right)=(11,2,0)$. The third prolongation is (also) of dimension 14 . The fourth tableau (no torsion) has characters $\left(s_{1}, s_{2}, s_{3}\right)=(12,2,0)$, and the fourth prolongation is of dimension 16. Thus the system is involutive by Cartan's test.

The Maurer-Cartan form (the components of non-negative degree) is given below. In degree 0:

$$
\begin{aligned}
\omega_{1}^{1} & =f_{1} \alpha^{1}+f_{2} \alpha^{2}+p_{1} \alpha^{3}, \quad \omega_{2}^{2}=g_{1} \alpha^{1}+g_{2} \alpha^{2}+p_{2} \alpha^{3}, \quad \omega_{2}^{1}=\alpha^{2}, \quad \omega_{1}^{2}=\alpha^{1} \\
\omega_{6}^{5} & =\alpha^{2}+\frac{1}{2}\left(2 f_{1}-g_{1}\right) \alpha^{3}, \quad \omega_{5}^{6}=\alpha^{1}+\frac{1}{2}\left(f_{2}-2 g_{1}\right) \alpha^{3} \\
0 & =\omega_{0}^{0}-\omega_{1}^{1}-\omega_{2}^{2}=\omega_{1}^{1}+\omega_{6}^{6}=\omega_{2}^{2}+\omega_{5}^{5}=\omega_{3}^{3}=\omega_{4}^{3}=\omega_{3}^{4}=\omega_{4}^{4}=\omega_{0}^{0}+\omega_{7}^{7} .
\end{aligned}
$$

The $f_{j}, g_{j}, 1 \leq j \leq 2$, are functions on the frame-bundle; the $f_{j, a}$ and $g_{j, a}$ are the $\alpha^{a}$-coefficients of $\mathrm{d} f_{j}$ and $\mathrm{d} g_{j}$; and $\mathrm{d} f_{1} \equiv \beta_{1}, \mathrm{~d} f_{2} \equiv-2 \beta_{2}, \mathrm{~d} g_{1} \equiv-2 \beta_{1}$ and 
$\mathrm{d} g_{2} \equiv \beta_{2}$ mod the semi-basic $\alpha^{a}$. Finally $p_{1}=-1+f_{1}\left(f_{2}-g_{2}\right)+f_{2,1}-f_{1,2}$ and $p_{2}=1+\left(f_{1}-g_{1}\right) g_{2}+g_{2,1}-g_{1,2}$. In degree 1 :

$$
\begin{array}{lll}
\omega_{3}^{1}=-\frac{1}{4}\left(2 f_{1}-g_{1}\right) \alpha^{2}-\beta_{2} & \omega_{4}^{1}=-\frac{9}{8}\left(2 f_{1}-g_{1}\right) \alpha^{2}-\frac{3}{2} \beta_{2} & \omega_{5}^{3}=-\frac{1}{8}\left(f_{2}-2 g_{2}\right) \alpha^{1}+p_{3} \alpha^{3}-\frac{1}{2} \beta_{1} \\
\omega_{4}^{2}=\frac{9}{8}\left(f_{2}-2 g_{2}\right) \alpha^{1}-\frac{3}{2} \beta_{1} & \omega_{3}^{2}=-\frac{1}{4}\left(f_{2}-2 g_{2}\right) \alpha^{1}+\beta_{1} & \omega_{6}^{3}=\frac{1}{8}\left(2 f_{1}-g_{1}\right) \alpha^{2}-p_{4} \alpha^{3}-\frac{1}{2} \beta_{2} \\
\omega_{5}^{4}=-\frac{1}{4}\left(f_{2}-2 g_{2}\right) \alpha^{1}+\beta_{1} & \omega_{6}^{4}=-\frac{1}{4}\left(2 f_{1}-g_{1}\right) \alpha^{2}-\beta_{2} & \omega_{7}^{5}=p_{4} \alpha^{3}-\beta_{2} \quad \omega_{7}^{6}=-p_{3} \alpha^{3}+\beta_{1} .
\end{array}
$$

Above, $p_{3}=\frac{1}{4}\left(2 f_{1}-g_{1}\right)+\frac{1}{2} g_{2}\left(f_{2}-2 g_{2}\right)+\frac{1}{4}\left(f_{2,2}-2 g_{2,2}\right)$ and $p_{4}=\frac{1}{4}\left(f_{2}-2 g_{2}\right)+$ $\frac{1}{2} f_{1}\left(2 f_{1}-g_{1}\right)+\frac{1}{4}\left(2 f_{1,1}-g_{1,1}\right)$. In degree 2 :

$$
\begin{gathered}
\omega_{4}^{0}=-\frac{3}{2} p_{3} \alpha^{1}+\frac{3}{2} p_{4} \alpha^{2}+h_{1} \alpha^{3}, \quad \omega_{7}^{3}=\frac{1}{2} p_{3} \alpha^{1}+\frac{1}{2} p_{4} \alpha^{2}+h_{2} \alpha^{3}-\frac{1}{2} \beta_{3}, \quad \omega_{7}^{4}=-\frac{2}{3} h_{1} \alpha^{3} \\
\omega_{5}^{1}=\frac{1}{4}\left(2 f_{1}-g_{1}+2 p_{3}\right) \alpha^{1}-\frac{1}{4}\left(f_{2}-2 g_{2}+2 p_{4}\right) \alpha^{2}+h_{1} \alpha^{3}-\frac{1}{2} \beta_{3} \\
\omega_{6}^{1}=\frac{1}{4}\left(f_{2}-2 g_{2}\right) \alpha^{1}-\left(2 p_{1}+p_{2}\right) \alpha^{2}+p_{5} \alpha^{3}-\beta_{1}, \quad \omega_{5}^{2}=\left(p_{1}-2 p_{2}\right) \alpha^{1}+\frac{1}{4}\left(2 f_{1}-g_{1}\right) \alpha^{2}+p_{6} \alpha^{3}+\beta_{2} \\
\omega_{6}^{2}=-\frac{1}{4}\left(2 f_{1}-g_{1}+2 p_{3}\right) \alpha^{1}+\frac{1}{4}\left(2 f_{2}-g_{2}+2 p_{4}\right) \alpha^{2}+h_{1} \alpha^{3}-\frac{1}{2} \beta_{3} .
\end{gathered}
$$

In degree 3 :

$$
\begin{aligned}
& \omega_{5}^{0}=2 p_{6} \alpha^{1}+\left(\frac{8}{3} h_{1}+\frac{3}{16}\left(2 f_{1}-g_{1}\right)\left(f_{2}-2 g_{2}\right)\right) \alpha^{2}+p_{7} \alpha^{3}-\frac{3}{4}\left(f_{2}-2 g_{2}\right) \beta_{2} \\
& \omega_{6}^{0}=\left(\frac{2}{3} h_{1}-\frac{3}{16}\left(2 f_{1}-g_{1}\right)\left(f_{2}-2 g_{2}\right)\right) \alpha^{1}-2 p_{5} \alpha^{2}+p_{8} \alpha^{3}+\frac{3}{4}\left(2 f_{1}-g_{1}\right) \beta_{1} \\
& \omega_{7}^{1}=\left(\frac{2}{3} h_{1}+\frac{1}{16}\left(2 f_{1}-g_{1}\right)\left(f_{2}-2 g_{2}\right)\right) \alpha^{1}+p_{5} \alpha^{2}+p_{9} \alpha^{3}-\frac{1}{4}\left(2 f_{1}-g_{1}\right) \beta_{1} \\
& \omega_{7}^{2}=p_{6} \alpha^{1}+\left(\frac{4}{3} h_{1}+\frac{1}{16}\left(2 f_{1}-g_{1}\right)\left(f_{2}-2 g_{2}\right)\right) \alpha^{2}+p_{10} \alpha^{3}+\frac{1}{4}\left(f_{2}-2 g_{2}\right) \beta_{2}
\end{aligned}
$$

The $p_{7}, \ldots, p_{10}$ above are polynomials in $\left\{f_{j}, g_{j}, h_{j}, f_{j, k}, g_{j, k}, h_{j, k}\right\}_{1 \leq j, k \leq 2}$. Finally, in degree 4 :

$\omega_{7}^{0}=p_{11} \alpha^{1}+p_{12} \alpha^{2}+\left(\frac{1}{2} h_{2,3}+\left(p_{1}+p_{2}\right) h_{2}\right) \alpha^{3}-\frac{1}{4}\left(f_{2}-2 g_{2}-8 p_{4}\right) \beta_{1}+\frac{1}{4}\left(2 f_{1}-g_{1}+4 p_{3}\right) \beta_{2}$.

The $p_{11}, p_{12}$ above are polynomials in the $\left\{f_{j}, g_{j}, h_{j}, f_{j, k}, g_{j, k}, h_{j, k}\right\}_{1 \leq j, k \leq 2}$.

Finally, we remark that the functions $f_{j}, g_{j}, h_{j}$ satisfy a system of partial differential equations. For example, $\left(f_{2}+2 g_{2}\right)_{1}=\left(2 f_{1}+g_{1}\right)_{2}$. One should think of this system as reducing the freedom of our initial data from four functions of 14 variables to the two functions of two variables and twelve functions of one variable and specified by the characters.

The Fubini cubics are

$$
\begin{aligned}
& r^{4}=-2\left(\alpha_{1}{ }^{3}+\alpha_{2}{ }^{3}\right)-\frac{4}{3} h_{1} \alpha_{3}{ }^{3} \\
& r^{5}=3 \alpha_{1}{ }^{2} \alpha_{2}+\frac{3}{2}\left(2 f_{1}-g_{1}\right) \alpha_{2} \alpha_{3}{ }^{2}+2 p_{4} \alpha_{3}{ }^{3} \\
& r^{6}=-3 \alpha_{1} \alpha_{2}{ }^{2}+\frac{3}{2}\left(f_{2}-2 g_{2}\right) \alpha_{1} \alpha_{3}{ }^{2}-2 p_{3} \alpha_{3}{ }^{3} \\
& r^{7}=0 .
\end{aligned}
$$

In $r^{4}, h_{1}$ is the term that first appeared in $\omega_{4}^{0}$ above.

The intrinsic invariants (cf. §9.4) are

$$
\begin{aligned}
& J_{1}=-\frac{1}{8}\left(2 f_{1}-g_{1}\right)-\frac{1}{2}\left(3 f_{2}-g_{2}\right) g_{2}-\frac{1}{4} f_{2,2}+\frac{4}{3} p_{3} \\
& J_{2}=-\frac{1}{8}\left(f_{2}-2 g_{2}\right)-\frac{1}{2} f_{1}\left(f_{1}+2 g_{1}\right)-\frac{1}{2} f_{1,1}-\frac{1}{3} p_{4} .
\end{aligned}
$$

9.4. Intrinsic invariants. Let $Y^{3} \subset \mathbb{P}^{7}$ be a 3 -fold with frame-bundle $\mathcal{F}_{\text {neg }}$ on which the Maurer-Cartan form is as given in (9.1). From (9.2) we see that $\mathbb{P}\{v \in$ $\left.T_{x} X \mid F_{2}(v, v)=0\right\} \subset \mathbb{P} T_{-1, x}$ consists of exactly two points. These two points define a pair of line bundles which span the contact hyperplane $T_{-1, x} \subset T_{x} X$.

Let's consider the situation more generally. Let $M$ be a complex 3-dimensional manifold (not necessarily projective) admitting two line bundles $L_{1}$ and $L_{2}$ such that $H:=L_{1} \oplus L_{2}$ is a contact distribution. Consider the bundle $\mathcal{F} \rightarrow M$ of all frames 
$\left\{e_{1}, e_{2}, e_{3}\right\}$ of $T_{x} M$ such that $e_{j}$ spans $L_{j}$, and given any local section $e: U \subset M \rightarrow \mathcal{F}$, $\left[e_{1}(x), e_{2}(x)\right] \equiv e_{3}(x) \bmod H_{x}$. Then $\mathcal{F}$ is a principle $G$-bundle with fibre group $G \subset \mathrm{GL}_{3} \mathbb{C}$

$$
G:=\left\{\left(\begin{array}{ccc}
a & 0 & \alpha \\
0 & b & \beta \\
0 & 0 & a b
\end{array}\right) \mid \begin{array}{l}
a, b \in \mathbb{C}, \\
\alpha, \beta \in \mathbb{C} \backslash\{0\}
\end{array}\right\} .
$$

Given $e=\left\{e_{j}\right\} \in \mathcal{F}_{x}$ and $v \in T_{e} \mathcal{F}$, the canonical semi-basic $\mathbb{C}^{3}$-valued 1-form on $\mathcal{F}$ is given by $\pi_{*}(v)=\eta^{j}(v) e_{j}$. There exist connection 1 -forms $\left\{\theta^{s}\right\}_{s=1}^{4}$ on $\mathcal{F}$ such that

$$
\begin{aligned}
& \mathrm{d} \eta^{1}=\eta^{1} \wedge \theta^{1}+\eta^{3} \wedge \theta^{3} \\
& \mathrm{~d} \eta^{2}=\eta^{2} \wedge \theta^{2}+\eta^{3} \wedge \theta^{4} \\
& \mathrm{~d} \eta^{3}=-\eta^{1} \wedge \eta^{2}+\eta^{3} \wedge\left(\theta^{1}+\theta^{2}\right) .
\end{aligned}
$$

Notice that this connection has torsion (see $\mathrm{d} \eta^{3}$ ). In fact, there are no torsion-free connections on $\mathcal{F}$. The family of connections preserving the structure equations above is 4-dimensional. so that

The choice of connection may be further refined (leaving one degree of freedom)

$$
\begin{aligned}
& \mathrm{d} \theta^{1}=2 \theta^{4} \wedge \eta^{1}+\theta^{3} \wedge \eta^{2}-\phi \wedge \eta^{3} \\
& \mathrm{~d} \theta^{2}=-\theta^{4} \wedge \eta^{1}-2 \theta^{3} \wedge \eta^{2}-\phi \wedge \eta^{3} \\
& \mathrm{~d} \theta^{3}=\theta^{2} \wedge \theta^{3}-\phi \wedge \eta^{1}+J_{1} \eta^{2} \wedge \eta^{3} \\
& \mathrm{~d} \theta^{4}=\theta^{1} \wedge \theta^{4}-\phi \wedge \eta^{2}+J_{2} \eta^{1} \wedge \eta^{3} .
\end{aligned}
$$

Above, $\phi$ is a 1 -form, and the $J$ are functions on $\mathcal{F}$.

The functions $J$ are relative invariants. They define invariant tensors $J_{1}\left(\omega^{2} \wedge\right.$ $\left.\omega^{3}\right) \otimes \underline{e}^{3}, J_{2}\left(\omega^{1} \wedge \omega^{3}\right) \otimes \underline{e}^{3} \in \Gamma\left(M, \bigwedge^{2}\left(T^{*} M\right) \otimes(T M / H)^{*}\right)$.

Applying the above formulas to Case 2 of 9.2 shows the two invariants $J_{1}$ and $J_{2}$ are zero, so the integral manifolds are intrinsically flat. Similarly, we see Case 3 $(\S 9.3)$ is not intrinsically flat.

9.5. Order three rigidity of $\boldsymbol{Z}_{\mathrm{ad}}^{\boldsymbol{A}_{\mathbf{2}}}$. It is also true that $I_{0} \subset I_{\mathrm{Fub}_{3}}$ but this is only seen to hold after one calculates a derivative. One can either prove third order rigidity of $X_{\mathrm{ad}}^{A_{2}}$ this way or cite [23]. 


\section{Remaining rigidity proofs.}

10.1. The varieties $v_{d}\left(\mathbb{P}^{n}\right), v_{d}\left(Q^{n}\right)$ and $\operatorname{Seg}\left(\mathbb{P}^{1} \times \mathbb{P}^{a_{2}} \times \cdots \times \mathbb{P}^{a_{n}}\right)$. Assume throughout this section that $n>1$.

Proof of Theorem 1.3 (a). Begin with the Veronese variety, and consider the Fubini system of order $d+1$. We will show it is a priori more restrictive than the $\left(I_{0}, J_{0}\right)$ system; thus the $\left(I_{0}^{\mathrm{f}}, \Omega\right)$ system being rigid implies it is rigid. Here $N_{k} \simeq S^{k} T^{*}$, the $F_{k, k}$ are the identity maps for $k \leq d$ and all the $F_{s, k}=0$ for $s<k$ and $F_{q}=0$ for $q>d$. Since the grading is three step, the osculating filtration coincides with the Lie algebra filtration (albeit with different integers attached to the filtrands).

The vanishing of the $F_{k, k+1}, k=2, \ldots, d$ fixes $N_{k}^{*} \otimes N_{k}$ in terms of $T^{*} \otimes T$ and $L^{*} \otimes L$, thus together they fix $\mathfrak{g l}(U)_{0}$ to be $\mathfrak{g}_{0}$. The vanishing of the $F_{k-1, k+1}, k=$ $3, \ldots, d$ fixes $N_{k}^{*} \otimes N_{k-1}$ in terms of $L^{*} \otimes T$ and the remaining forms in the system fix all other components of the Maurer-Cartan form taking values in the remaining spaces below the diagonal to be zero. Thus we are reduced to the $\left(I_{0}, J_{0}\right)$ system, and Theorem 1.3 (a) follows from Theorem 1.9.

Remark. Note that we only used a small part of the Fubini system to prove rigidity in this case.

Remark. In in the case $d=2, n=1, v_{2}\left(\mathbb{P}^{1}\right) \subset \mathbb{P}^{2}$ is a plane conic. Monge showed that the plane conics are rigid at order 5 . This is consistent with the Lie algebra cohomology: In this case we have $\mathfrak{g}=U=S^{2} \mathbb{C}^{2}=U_{2 \omega_{1}}$. The decomposition $\mathfrak{s l}(U)=U_{2 \omega_{1}} \oplus U_{4 \omega_{1}}$ yields $\mathfrak{g}^{\perp}=U_{4 \omega_{1}}$. The cohomology group $H^{1}\left(\mathfrak{g}_{-}, \mathfrak{g}^{\perp}\right)$ is onedimensional, with weight $-6 \omega_{1}$ as a $\mathfrak{g}_{0}=\mathbb{C}$ module. Since the grading element $Z$ for $U$ is given by $Z\left(\omega_{1}\right)=\frac{1}{2}$, we see that $H_{3}^{1}\left(\mathfrak{g}_{-}, \mathfrak{g}^{\perp}\right) \neq 0$, an obstruction to Fubini rigidity at order four.

Proof of Theorem $1.3(b)$. The $v_{d}(Q)$ Fubini system is similar to the Veronese system. The grading is again 3-step. All Fubini forms are zero except for the fundamental forms and they are given by $N_{k} \simeq S^{k} T^{*}$ for $k \leq d, N_{d+1}=Q \circ S^{d-1} T^{*}$, $N_{d+2}=Q^{2} \circ S^{d-2} T^{*}, \ldots, N_{2 d}=Q^{d}$ and all higher fundamental forms are zero. Here $F_{2 d+1,2 d}$ fixes the $N_{d}^{*} \otimes N_{d}$ component of the Maurer-Cartan form in terms of the $\left(T^{*} \otimes T\right) \oplus\left(L^{*} \otimes L\right)$-component, and similarly down the line. Thus Theorem 1.3 (b) follows from Theorem 7.2.

Proof of Theorem 1.3 (c). We merely sketch the proof for the Segre variety $\operatorname{Seg}\left(\mathbb{P}^{1} \times \mathbb{P}^{a_{2}} \times \cdots \times \mathbb{P}^{a_{r}}\right)-$ it is similar to the two above. The $r+1$ Fubini system implies the $\left(I_{0}, J_{0}\right)$ system. We compute that $H_{d}^{1}\left(\mathfrak{g}_{-}, \mathfrak{g}^{\perp}\right)=0$ for $d>1$. Thus the $\left(I_{0}, J_{0}\right)$ system is rigid.

10.2. $\boldsymbol{E}_{\mathbf{8}} / \boldsymbol{P}_{\mathbf{1}} \subset \mathbb{P}^{\mathbf{3 8 7 4}}$. To establish that $E_{8} / P_{1}$ is rigid to order five, it suffices to show that the osculating sequence has length four and that the $\omega_{\mathfrak{g}_{\llcorner 0}^{\perp}}$ component of the Maurer-Cartan form is forced to vanish on integral manifolds of the fifth order Fubini system. The first assertion follows because the shortest sequence of negative roots taking $\omega_{1}$ to $-\omega_{1}$ is of length four. The second by arguments similar to the adjoint case for the $T_{-1}^{*} \otimes T_{-1}$ component, forcing this component of the MaurerCartan form to be the spin representation of $D_{7}$ and by observing that the fourth fundamental form is the quadric on $T_{-2}$, forcing the $T_{-2}^{*} \otimes T_{-2}$ component of the Maurer-Cartan form to be the standard representation of $D_{7}$. 


\section{REFERENCES}

[1] N. Bourbaki, Groupes et algèbres de Lie, Hermann, Paris, 1968, MR0682756.

[2] R. L. Bryant, S.-S. Chern, R. B. Gardner, H. L. Goldschmidt and P. A. Griffiths, Exterior differential systems, Mathematical Sciences Research Institute Publications, 18. Springer-Verlag, New York, 1991. viii+475.

[3] R. L. BRyAnt, Rigidity and quasi-rigidity of extremal cycles in Hermitian symmetric spaces, Princeton University Press, AM-153, 2005.

[4] A. Cap And H. Schichl, Parabolic geometries and canonical Cartan connections, Hokkaido Math. J., 29 (2000), pp. 453-505.

[5] A. CAP, Differential operators and overdetermined systems, preprint.

[6] E. CARTAN, Sur les variétés a connexion projective (French), Bull. Soc. Math. France, 52 (1924), pp. 205-241.

[7] E. CARTAN, Sur les variétés de courbure constante d'un espace euclidien ou non euclidien, Bull. Soc. Math France, 47 (1919), pp. 125-160; and 48 (1920), pp. 132-208; see also pp. 321-432 in Oeuvres Complètes Part 3, Gauthier-Villars, 1955.

[8] G. Fubini, Studi relativi all'elemento lineare proiettivo di una ipersuperficie, Rend. Acad. Naz. dei Lincei, 1918, pp. 99-106.

[9] P. A. Griffiths and J. Harris, Algebraic Geometry and Local Differential Geometry, Ann. scient. Ec. Norm. Sup., 12 (1979), pp. 355-432, MR0559347.

[10] J.-M. HWANG AND K. YAMAguCHI, Characterization of Hermitian symmetric spaces by fundamental forms, Duke Math. J., 120 (2003), pp. 621-634.

[11] T. IVEy AND J. M. LANDSBERG, Cartan for beginners: differential geometry via moving frames and exterior differential systems, Graduate Studies in Mathematics, 61, American Mathematical Society, Providence, RI, 2003, MR2003610.

[12] J. Hong, Rigidity of smooth Schubert varieties in Hermitian symmetric spaces, Trans. Amer. Math. Soc., 359 (2007), pp. 2361-2381.

[13] J. Hong, Rigidity of singular Schubert varieties in $\operatorname{Gr}(m, n)$, J. Differential Geom., 71 (2005), pp. $1-22$.

[14] B. Kostant, Lie algebra cohomology and the generalized Borel-Weil theorem, Ann. of Math. (2), 74 (1961), pp. 329-387, MR0142696.

[15] J. M. Landsberg, Differential-geometric characterizations of complete intersections, J. Differential Geom., 44 (1996), pp. 32-73, MR1420349.

[16] J. M. LAndsberg, On the infinitesimal rigidity of homogeneous varieties, Compositio Math., 118 (1999), pp. 189-201, MR1713310.

[17] J. M. LANDSBerG, Griffiths-Harris rigidity of compact Hermitian symmetric spaces, J. Differential Geom., 74 (2006), pp. 395-405.

[18] J. M. LANDSBerg, Differential geometry of submanifolds of projective space, to appear in the proceedings of the 2006 IMA workshop "Symmetries and overdetermined systems of partial differential equations".

[19] J. M. LANDSBerg AND L. MANIVEL, On the projective geometry of rational homogeneous varieties, Comment. Math. Helv., 78(1) (2003), pp. 65-100, MR1966752.

[20] J. M. Landsberg and L. Manivel, Classification of simple Lie algebras via projective geometry, Selecta Mathematica, 8 (2002), pp. 137-159, MR1890196.

[21] J. M. Landsberg and L. Manivel, Series of Lie groups, Michigan Math. J., 52(2) (2004), pp. 453-479, MR2069810.

[22] J. M. Landsberg And L. Manivel, The projective geometry of Freudenthal's magic square, J. Algebra, 239 (2001), pp. 477-512.

[23] C. Robles, The adjoint variety of $\mathrm{SL}_{m+1} \mathbb{C}$ is rigid to order three, Differential Geom. Appl., 26 (2008), pp. 683-696.

[24] T. Sasaki, K. Yamaguchi And M. Yoshida, On the rigidity of differential systems modelled on Hermitian symmetric spaces and disproofs of a conjecture concerning modular interpretations of configuration spaces, in CR-geometry and overdetermined systems (Osaka, 1994), pp. 318-354, Adv. Stud. Pure Math., 25, Math. Soc. Japan, Tokyo, 1997.

[25] Y. SE-AsHi, On differential invariants of integrable finite type linear differential equations, Hokkaido Math. J., 17 (1988), pp. 151-195. MR0945853

[26] K. Yamaguchi, Differential systems associated with simple graded Lie algebras, Progress in differential geometry, pp. 413-494, Adv. Stud. Pure Math., 22, Math. Soc. Japan, Tokyo, 1993. 